

\title{
The radius of a subcategory of modules
}

\author{
Hailong Dao and Ryo Takahashi
}

\author{
Dedicated to Professor Craig Huneke on the occasion of his sixtieth birthday
}

\begin{abstract}
We introduce a new invariant for subcategories $\mathscr{X}$ of finitely generated modules over a local ring $R$ which we call the radius of $\mathscr{X}$. We show that if $R$ is a complete intersection and $\mathscr{X}$ is resolving, then finiteness of the radius forces $\mathscr{X}$ to contain only maximal Cohen-Macaulay modules. We also show that the category of maximal Cohen-Macaulay modules has finite radius when $R$ is a Cohen-Macaulay complete local ring with perfect coefficient field. We link the radius to many well-studied notions such as the dimension of the stable category of maximal Cohen-Macaulay modules, finite/countable Cohen-Macaulay representation type and the uniform Auslander condition.
\end{abstract}

\section{Introduction}

Let $R$ be a commutative Noetherian local ring and $\bmod R$ the category of finitely generated modules over $R$. In this paper we introduce and study a new invariant for subcategories $\mathscr{X}$ of mod $R$ which we call the radius of $\mathscr{X}$. Roughly speaking, it is defined as the least number of extensions necessary to build the whole objects in $\mathscr{X}$ out of a single object in mod $R$. (For the precise definition, see Definition 2.3 in this paper.) Our definition is inspired by the notion of dimension of triangulated categories that was introduced by Rouquier [2008].

We obtain strong evidences that the concept of radius is intimately linked to both the representation theory and the singularity of $R$. For example, over a Gorenstein complete local ring $R$, the category of maximal Cohen-Macaulay modules has radius zero if and only if $R$ has finite Cohen-Macaulay representation type, in other words, $R$ is a simple hypersurface singularity (when $R$ has an algebraically closed coefficient field of characteristic zero). In addition, the category of maximal CohenMacaulay modules over a complete local hypersurface (over an algebraically closed

Dao was partially supported by NSF grant DMS 0834050. Takahashi was partially supported by JSPS Grant-in-Aid for Young Scientists (B) 22740008 and by JSPS Postdoctoral Fellowships for Research Abroad.

MSC2010: primary 13C60; secondary 13C14, 16G60, $18 \mathrm{E} 30$.

Keywords: radius of subcategory, resolving subcategory, thick subcategory, Cohen-Macaulay module, complete intersection, dimension of triangulated category, Cohen-Macaulay representation type. 
field of characteristic not two) of countable Cohen-Macaulay representation type has radius one. We also observe a tantalizing connection to the uniform Auslander condition, which has attracted researchers over the years.

Perhaps most surprisingly, one corollary of our first main result (Theorem 3.3) states:

Theorem I. Let $R$ be a local complete intersection, and let $\mathscr{X}$ be a resolving subcategory of $\bmod R$. If the radius of $\mathscr{X}$ is finite, then $\mathscr{X}$ contains only maximal Cohen-Macaulay modules.

We conjecture that the above result holds for all Cohen-Macaulay local rings. Our second main result below supports this conjecture, which follows from more general results (Theorems 5.7 and 5.11).

Theorem II. Let $R$ be a Cohen-Macaulay complete local ring with perfect coefficient field. Then the category of maximal Cohen-Macaulay modules over $R$ has finite radius.

The structure of the paper is as follows. In Section 1 we set the basic notations and definitions. Section 2 contains our key definition (Definition 2.1) of the radius of a subcategory of $\bmod R$, as well as some detailed comparisons to similar notions. We also give several results connecting the radius to the singularities of finite and countable Cohen-Macaulay representation type. Sections 3 and 4 consist of the statement and proof of our Theorem I, respectively. We also discuss here thickness of resolving subcategories of maximal Cohen-Macaulay modules over a complete intersection. Section 5 contains the proof of (generalizations of) our Theorem II. Section 6 connects the main results to the uniform Auslander condition and discusses some open questions.

\section{Preliminaries}

In this section, we recall the definitions of a resolving subcategory, totally reflexive modules and a thick subcategory.

Convention 1.1. Throughout this paper, we assume all rings are commutative Noetherian rings with identity. All modules are finitely generated. All subcategories are full and strict. (Recall that a subcategory $\mathscr{X}$ of a category $\mathscr{C}$ is called strict provided that for objects $M, N \in \mathscr{C}$ with $M \cong N$, if $M$ is in $\mathscr{X}$, then so is $N$.) Hence, the subcategory of a category $\mathscr{C}$ consisting of objects $\left\{M_{\lambda}\right\}_{\lambda \in \Lambda}$ always means the smallest strict full subcategory of $\mathscr{C}$ to which $M_{\lambda}$ belongs for all $\lambda \in \Lambda$. Note that this coincides with the full subcategory of $\mathscr{C}$ consisting of all objects $X \in \mathscr{C}$ such that $X \cong M_{\lambda}$ for some $\lambda \in \Lambda$. Let $R$ be a (commutative Noetherian) ring. Denote by $\bmod R$ the category of (finitely generated) $R$-modules and $R$-homomorphisms. For a Cohen-Macaulay local ring $R$, we call a maximal Cohen-Macaulay $R$-module 
just a Cohen-Macaulay $R$-module. We denote by $\mathrm{CM}(R)$ the subcategory of $\bmod R$ consisting of Cohen-Macaulay $R$-modules.

The following notation is used throughout this paper.

Notation 1.2. For a subcategory $\mathscr{X}$ of $\bmod R$, we denote by add $\mathscr{X}\left(\right.$ or $\left.\operatorname{add}_{R} \mathscr{L}\right)$ the additive closure of $\mathscr{X}$, namely, the subcategory of $\bmod R$ consisting of direct summands of finite direct sums of modules in $\mathscr{X}$. When $\mathscr{X}$ consists of a single module $M$, we simply denote it by add $M\left(\right.$ or $\left.\operatorname{add}_{R} M\right)$. For an $R$-module $M$, we denote by $M^{*}$ the $R$-dual module $\operatorname{Hom}_{R}(M, R)$. For a homomorphism $f: M \rightarrow N$ of $R$-modules, $f^{*}$ denotes the $R$-dual homomophism $N^{*} \rightarrow M^{*}$ sending $\sigma \in N^{*}$ to the composition $\sigma \cdot f \in M^{*}$.

The notion of a resolving subcategory has been introduced by Auslander and Bridger [1969]. It can actually be defined for an arbitrary abelian category with enough projective object. The only resolving subcategories we deal with in this paper are ones of $\bmod R$.

Definition 1.3. A subcategory $\mathscr{L}$ of $\bmod R$ is called resolving if the following hold.

(R1) $\mathscr{X}$ contains the projective $R$-modules.

(R2) $\mathscr{X}$ is closed under direct summands: if $M$ is an $R$-module in $\mathscr{X}$ and $N$ is an $R$-module that is a direct summand of $M$, then $N$ is also in $\mathscr{X}$.

(R3) $\mathscr{X}$ is closed under extensions: for an exact sequence $0 \rightarrow L \rightarrow M \rightarrow N \rightarrow 0$ of $R$-modules, if $L, N$ are in $\mathscr{X}$, then so is $M$.

(R4) $\mathscr{X}$ is closed under kernels of epimorphisms: for an exact sequence $0 \rightarrow L \rightarrow$ $M \rightarrow N \rightarrow 0$ of $R$-modules, if $M, N$ are in $\mathscr{X}$, then so is $L$.

A resolving subcategory is a subcategory such that any two minimal resolutions of a module by modules in it have the same length; see [Auslander and Bridger 1969, Lemma (3.12)]. Note that one can replace the condition (R1) with:

$\left(\mathrm{R} 1^{\prime}\right) \mathscr{X}$ contains $R$.

Next we recall the notion of a totally reflexive module.

Definition 1.4. An $R$-module $M$ is called totally reflexive if the natural homomorphism $M \rightarrow M^{* *}$ is an isomorphism and $\operatorname{Ext}_{R}^{i}(M, R)=0=\operatorname{Ext}_{R}^{i}\left(M^{*}, R\right)$ for all $i>0$. We denote by $\mathscr{G}(R)$ the subcategory of $\bmod R$ consisting of totally reflexive modules.

A totally reflexive module was defined by Auslander [1967], and deeply studied by Auslander and Bridger [1969]. The $R$-dual of a totally reflexive $R$-module is also totally reflexive. Every projective module is totally reflexive, i.e., add $R \subseteq \mathscr{G}(R)$. If $R$ is a Cohen-Macaulay local ring, then every totally reflexive $R$-module is CohenMacaulay, i.e., $\mathscr{G}(R) \subseteq \mathrm{CM}(R)$. When $R$ is a Gorenstein local ring, an $R$-module 
is totally reflexive if and only if it is Cohen-Macaulay, i.e., $\mathscr{G}(R)=\mathrm{CM}(R)$. For more details, see [Auslander and Bridger 1969] and [Christensen 2000].

Syzygies, cosyzygies and transposes are key tools in this paper. We recall here their precise definitions.

Definition 1.5. Let $(R, \mathfrak{m})$ be a local ring, and let $M$ be an $R$-module.

(1) Take a minimal free resolution $\cdots \stackrel{\delta_{n+1}}{\longrightarrow} F_{n} \stackrel{\delta_{n}}{\rightarrow} F_{n-1} \stackrel{\delta_{n-1}}{\longrightarrow} \cdots \stackrel{\delta_{1}}{\rightarrow} F_{0} \rightarrow M \rightarrow 0$ of $M$. Then, for each $n \geq 1$, the image of $\delta_{n}$ is called the $n$-th syzygy of $M$ and denoted by $\Omega^{n} M$ (or $\Omega_{R}^{n} M$ ). For convention, we set $\Omega^{0} M=M$.

(2) The cokernel of the $R$-dual map $\delta_{1}^{*}: F_{0}^{*} \rightarrow F_{1}^{*}$ is called the (Auslander) transpose of $M$ and denoted by $\operatorname{Tr} M$ (or $\operatorname{Tr}_{R} M$ ).

(3) Let $0 \rightarrow M \rightarrow F_{-1} \stackrel{\delta_{-1}}{\longrightarrow} \cdots \stackrel{\delta_{-(n-1)}}{\longrightarrow} F_{-n} \stackrel{\delta_{-n}}{\longrightarrow} F_{-(n+1)} \stackrel{\delta_{-(n+1)}}{\longrightarrow} \cdots$ be a minimal free coresolution of $M$, that is, an exact sequence with $F_{-n}$ free and $\operatorname{Im} \delta_{-n} \subseteq$ $\mathfrak{m} F_{-(n+1)}$ for all $n \geq 1$. Then we call the image of $\delta_{-n}$ the $n$-th cosyzygy of $M$ and denote it by $\Omega^{-n} M$ (or $\Omega_{R}^{-n} M$ ).

Let $R$ be a local ring. Then by [Yoshino 2005, Lemma 3.2] one can replace (R4) with:

(R4') $\mathscr{X}$ is closed under syzygies: if $M$ is in $\mathscr{X}$, then so is $\Omega M$.

Totally reflexive modules behave well under taking their syzygies, cosyzygies and transposes. Let $R$ be a local ring. Let $M$ be a totally reflexive $R$-module. The $R$-dual of a minimal free resolution (respectively, coresolution) of $M$ is a minimal free coresolution (respectively, resolution) of $M^{*}$. In particular, a minimal free coresolution of $M$ always exists, and it is uniquely determined up to isomorphism. The $n$-th syzygy $\Omega^{n} M$ and cosyzygy $\Omega^{-n} M$ are again totally reflexive for all $n$. This is an easy consequence of [Christensen 2000, (1.2.9) and (1.4.8)]. The transpose $\operatorname{Tr} M$ is also totally reflexive; see [Auslander and Bridger 1969, Proposition (3.8)]. For an $R$-module $M$, the $n$-th syzygy $\Omega^{n} M$ for any $n \geq 1$ and the transpose $\operatorname{Tr} M$ are uniquely determined up to isomorphism, since so is a minimal free resolution of $M$. If $M$ is totally reflexive, then the $n$-th cosyzygy $\Omega^{-n} M$ for any $n \geq 1$ is also uniquely determined up to isomorphism, since so is a minimal free coresolution of $M$.

A lot of subcategories of mod $R$ are known to be resolving. For example, $\operatorname{CM}(R)$ is a resolving subcategory of $\bmod R$ if $R$ is Cohen-Macaulay. The subcategory of $\bmod R$ consisting of totally reflexive $R$-modules is resolving by [Auslander and Bridger 1969, (3.11)]. One can construct a resolving subcategory easily by using the vanishing of Tor or Ext. Also, the modules of complexity less than a fixed integer form a resolving subcategory of $\bmod R$. For the details, we refer to [Takahashi 2009, Example 2.4].

Now we define a thick subcategory of totally reflexive modules. 
Definition 1.6. A subcategory $\mathscr{X}$ of $\mathscr{G}(R)$ is called thick if it is closed under direct summands and short exact sequences: for an exact sequence $0 \rightarrow L \rightarrow M \rightarrow N \rightarrow 0$ of totally reflexive $R$-modules, if two of $L, M, N$ are in $\mathscr{X}$, then so is the third.

A typical example of a thick subcategory is obtained by restricting a resolving subcategory to $\mathscr{G}(R)$.

The following proposition is shown by an argument dual to [Yoshino 2005, Lemma 3.2].

Proposition 1.7. Let $R$ be a local ring. Let $\mathscr{X}$ be a subcategory of $\mathscr{G}(R)$ containing $R$. Then $\mathscr{X}$ is a thick subcategory of $\mathscr{G}(R)$ if and only if $\mathscr{X}$ is a resolving subcategory of $\bmod R$ and is closed under cosyzygies: if $M$ is in $\mathscr{X}$, then so is $\Omega^{-1} M$.

Let $(R, \mathfrak{m})$ be a local ring. We call $R$ a hypersurface if the $\mathfrak{m}$-adic completion $\widehat{R}$ of $R$ is a residue ring of a complete regular local ring by a principal ideal. We say that $R$ is a complete intersection if $\widehat{R}$ is a residue ring of a complete regular local ring by an ideal generated by a regular sequence.

We recall the definitions of Gorenstein dimension and complete intersection dimension, which are abbreviated to G-dimension and CI-dimension. These notions have been introduced by Auslander and Bridger [1969] and Avramov, Gasharov and Peeva [Avramov et al. 1997], respectively.

Definition 1.8. Let $R$ be a local ring, and let $M$ be an $R$-module. The $G$-dimension of $M$, denoted $\operatorname{Gdim}_{R} M$, is defined as the infimum of the lengths of totally reflexive resolutions of $M$, namely, exact sequences of the form $0 \rightarrow X_{n} \rightarrow X_{n-1} \rightarrow \cdots \rightarrow$ $X_{1} \rightarrow X_{0} \rightarrow M \rightarrow 0$ with each $X_{i}$ being totally reflexive. The CI-dimension of $M$ is defined as the infimum of $\operatorname{pd}_{S}\left(M \otimes_{R} R^{\prime}\right)-\operatorname{pd}_{S} R_{f}^{\prime}$, where $R \rightarrow R^{\prime} \leftarrow S$ runs over the quasi-deformations of $R$. Here, a diagram $R \stackrel{f}{\rightarrow} R^{\prime} \stackrel{g}{\leftarrow} S$ of homomorphisms of local rings is called a quasi-deformation of $R$ if $f$ is faithfully flat and $g$ is a surjection whose kernel is generated by an $S$-sequence.

Recall that $M$ is said to have complexity $c$, denoted by $\mathrm{cx}_{R} M=c$, if $c$ is the least nonnegative integer $n$ such that there exists a real number $r$ satisfying the inequality $\beta_{i}^{R}(M) \leq r i^{n-1}$ for all $i \gg 0$.

Remark 1.9. For a local ring $(R, \mathfrak{m}, k)$ and a module $M$ over $R$, the following are known to hold. For the proofs, we refer to [Christensen 2000] and [Avramov et al. 1997].

(1) $\operatorname{Gdim}_{R} M=\infty$ if and only if $M$ does not admit a totally reflexive resolution of finite length.

(2) $\operatorname{Cldim}_{R} M=\infty$ if and only if $\operatorname{pd}_{S}\left(M \otimes_{R} R^{\prime}\right)=\infty$ for every quasi-deformation $R \rightarrow R^{\prime} \leftarrow S$.

(3) One has $M=0 \Leftrightarrow \operatorname{Gdim}_{R} M=-\infty \Leftrightarrow \operatorname{Cldim}_{R} M=-\infty$. 
(4) $\operatorname{Gdim}_{R} M \leq 0$ if and only if $M$ is totally reflexive.

(5) If $\operatorname{Gdim}_{R} M$ (respectively, $\operatorname{Cldim}_{R} M$ ) is finite, it is equal to depth $R-\operatorname{depth}_{R} M$.

(6) The inequalities $\operatorname{Gdim}_{R} M \leq \operatorname{Cldim}_{R} M \leq \operatorname{pd}_{R} M$ hold, and equalities hold to the left of any finite dimension.

(7) If $M \neq 0$, then $\operatorname{Gdim}_{R}\left(\Omega^{n} M\right)=\sup \left\{\operatorname{Gdim}_{R} M-n, 0\right\}$ and $\operatorname{Cldim}_{R}\left(\Omega^{n} M\right)=$ $\sup \left\{\operatorname{Cldim}_{R} M-n, 0\right\}$ hold for all $n \geq 0$.

(8) If $R$ is a Gorenstein ring (respectively, a complete intersection), then $\operatorname{Gdim}_{R} M$ (respectively, $\operatorname{Cldim}_{R} M$ ) is finite. If $\operatorname{Gdim}_{R} k$ (respectively, $\operatorname{Cldim}_{R} k$ ) is finite, then $R$ is a Gorenstein ring (respectively, a complete intersection).

(9) If $\operatorname{Cldim}_{R} M<\infty$, then $\mathrm{cx}_{R} M<\infty$.

\section{Definition of the radius of a subcategory}

This section contains the key definition and establishes several results. More precisely, we will give the definition of the radius of a subcategory of $\bmod R$ for a local ring $R$, and compare it with other notions, such as the dimension of a triangulated category defined by Rouquier. We will also explore its relationships with representation types of a Cohen-Macaulay local ring.

Definition 2.1. Let $R$ be a local ring.

(1) For a subcategory $\mathscr{X}$ of $\bmod R$ we denote by [Q⿻ the additive closure of the subcategory of $\bmod R$ consisting of $R$ and all modules of the form $\Omega^{i} X$, where $i \geq 0$ and $X \in \mathscr{X}$. When $\mathscr{X}$ consists of a single module $X$, we simply denote it by $[X]$.

(2) For subcategories $\mathscr{X}, \mathscr{Y}$ of $\bmod R$ we denote by $\mathscr{L} \circ \mathscr{Y}$ the subcategory of $\bmod R$ consisting of the $R$-modules $M$ which fits into an exact sequence $0 \rightarrow X \rightarrow M \rightarrow Y \rightarrow 0$ with $X \in \mathscr{X}$ and $Y \in \mathscr{Y}$. We set $\mathscr{L} \bullet \mathscr{Y}=[[\mathscr{X}] \circ[\mathscr{Y}]]$.

(3) Let $\mathscr{C}$ be a subcategory of $\bmod R$. We define the ball of radius $r$ centered at $\mathscr{C}$ as

$$
[\mathfrak{b}]_{r}= \begin{cases}{[\mathfrak{C}]} & \text { if } r=1, \\ {[\mathfrak{C}]_{r-1} \bullet \mathscr{C}=\left[[\mathscr{C}]_{r-1} \circ[\mathfrak{b}]\right]} & \text { if } r \geq 2 .\end{cases}
$$

If $\mathscr{C}$ consists of a single module $C$, then we simply denote $[\mathscr{C}]_{r}$ by $[C]_{r}$, and call it the ball of radius $r$ centered at $C$. We write $\left[\complement^{\ell}\right]_{r}^{R}$ when we should specify that $\bmod R$ is the ground category where the ball is defined.

Some similar notions have already been introduced. Takahashi [2009, Definition 3.1] defines the subcategory res ${ }^{n} \mathscr{L}$ of the resolving closure res $\mathscr{L}$ of a given subcategory $\mathscr{X}$ of $\bmod R$. This is different from ours in that res ${ }^{n} \mathscr{X}$ is not closed under syzygies. In [Avramov et al. 2010] the thickening thick ${ }^{n} \mathscr{L}$ of a given subcategory $\mathscr{X}$ of a triangulated category is defined. This cannot be applied directly to a module category. 
Proposition 2.2. Let $R$ be a local ring.

(1) Let $\mathscr{X}, 9$ be subcategories of $\bmod R$. The following are equivalent for an $R$-module $M$ :

(a) $M$ belongs to $\mathscr{X} \bullet Y$.

(b) There exists an exact sequence $0 \rightarrow X \rightarrow Z \rightarrow Y \rightarrow 0$ of $R$-modules with $X \in[\mathscr{X}]$ and $Y \in[\mathscr{Y}]$ such that $M$ is a direct summand of $Z$.

(2) For subcategories $\mathscr{X}, \mathscr{Y}, \mathscr{L}$ of $\bmod R$, one has $(\mathscr{X} \bullet Y) \bullet \mathscr{L}=\mathscr{X} \bullet(\mathscr{Y} \bullet \mathscr{L})$.

(3) Let $\mathscr{C}$ be a subcategory of $\bmod R$, and let $a, b$ be positive integers. Then one has $[\mathfrak{C}]_{a} \bullet[\mathfrak{C}]_{b}=[\mathfrak{C}]_{a+b}=[\mathfrak{C}]_{b} \bullet[\mathfrak{C}]_{a}$.

Proof. (1) The implication (b) $\Rightarrow$ (a) is obvious. To prove the opposite implication (a) $\Rightarrow$ (b), let $M$ be an $R$-module in $\mathscr{X} \bullet \mathcal{Y}=[[\mathscr{X}] \circ[\mathscr{Y}]]$. By definition, $M$ is isomorphic to a direct summand of $R^{\oplus p} \oplus \bigoplus_{i=0}^{n}\left(\Omega^{i} Z_{i}\right)^{\oplus q_{i}}$, where $p, q_{i} \geq 0$ and $Z_{i} \in[\mathscr{X}] \circ[Y]$. For each $0 \leq i \leq n$ there is an exact sequence $0 \rightarrow X_{i} \rightarrow Z_{i} \rightarrow Y_{i} \rightarrow 0$ with $X_{i} \in[\mathscr{X}]$ and $Y_{i} \in[\mathscr{Y}]$. Taking syzygies and direct sums, we have an exact sequence

$0 \rightarrow R^{\oplus p} \oplus \bigoplus_{i=0}^{n}\left(\Omega^{i} X_{i}\right)^{\oplus q_{i}} \rightarrow R^{\oplus p} \oplus \bigoplus_{i=0}^{n}\left(\Omega^{i} Z_{i}\right)^{\oplus q_{i}} \oplus R^{\oplus r} \rightarrow \bigoplus_{i=0}^{n}\left(\Omega^{i} Y_{i}\right)^{\oplus q_{i}} \rightarrow 0$.

The left and right terms are in [ஜ] and [Y], respectively. The middle term contains an $R$-module isomorphic to $M$. Thus the statement (b) follows.

(2) First, let $M$ be an $R$-module in $(\mathscr{C} \bullet \mathscr{Y}) \bullet \mathscr{Z}$. By the assertion (1) there is an exact sequence $0 \rightarrow W \stackrel{f}{\rightarrow} V \rightarrow Z \rightarrow 0$ with $W \in \mathscr{X} \bullet$ y and $Z \in[\mathscr{L}]$ such that $M$ is a direct summand of $V$. By (1) again, we have an exact sequence $0 \rightarrow X \rightarrow U \rightarrow Y \rightarrow 0$ with $X \in[\mathscr{X}]$ and $Y \in[\mathscr{Y}]$ such that $W$ is a direct summand of $U$. Writing $U=W \oplus W^{\prime}$, we make the following pushout diagram.

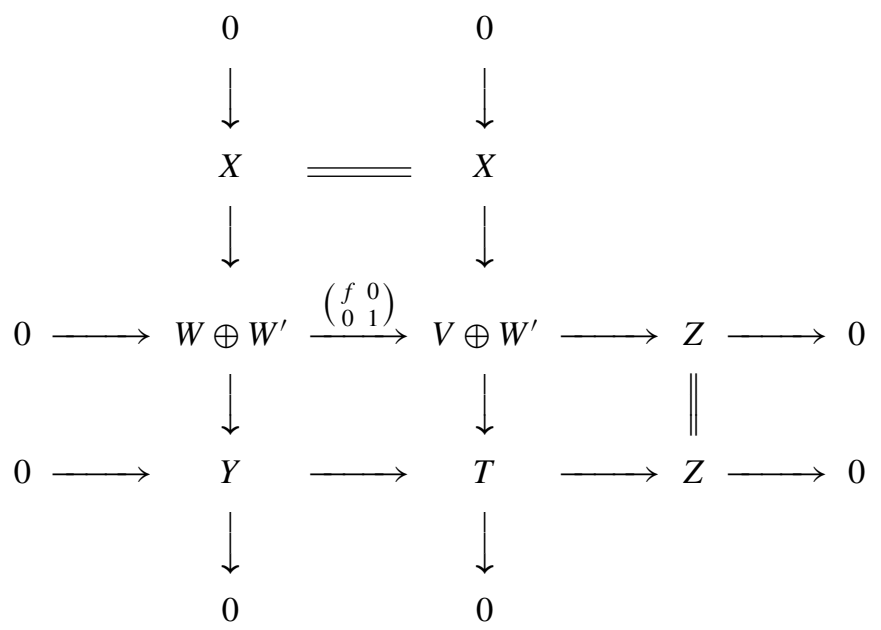


The bottom row implies that $T$ is in $\mathscr{Y} \bullet \mathscr{L}$, and it follows from the middle column that $M$ belongs to $\mathscr{X} \bullet(\mathscr{Y} \bullet \mathscr{L})$. Hence we have $(\mathscr{X} \bullet \mathcal{Y}) \bullet \mathscr{L} \subseteq \mathscr{X} \bullet(Y) \bullet \mathscr{L})$.

Next, let $M$ be an $R$-module in $\mathscr{X} \bullet(\mathscr{Y} \bullet \mathscr{L})$. Then it follows from (1) that there is an exact sequence $0 \rightarrow X \rightarrow V \stackrel{f}{\rightarrow} W \rightarrow 0$ with $X \in[\mathscr{X}]$ and $W \in \mathscr{Y} \bullet \mathscr{L}$ such that $M$ is a direct summand of $V$. Applying (1) again, we have an exact sequence $0 \rightarrow Y \rightarrow U \rightarrow Z \rightarrow 0$ with $Y \in[\mathscr{Y}]$ and $Z \in[\mathscr{Z}]$ such that $W$ is a direct summand of $U$. Write $U=W \oplus W^{\prime}$, and we have a pullback diagram:

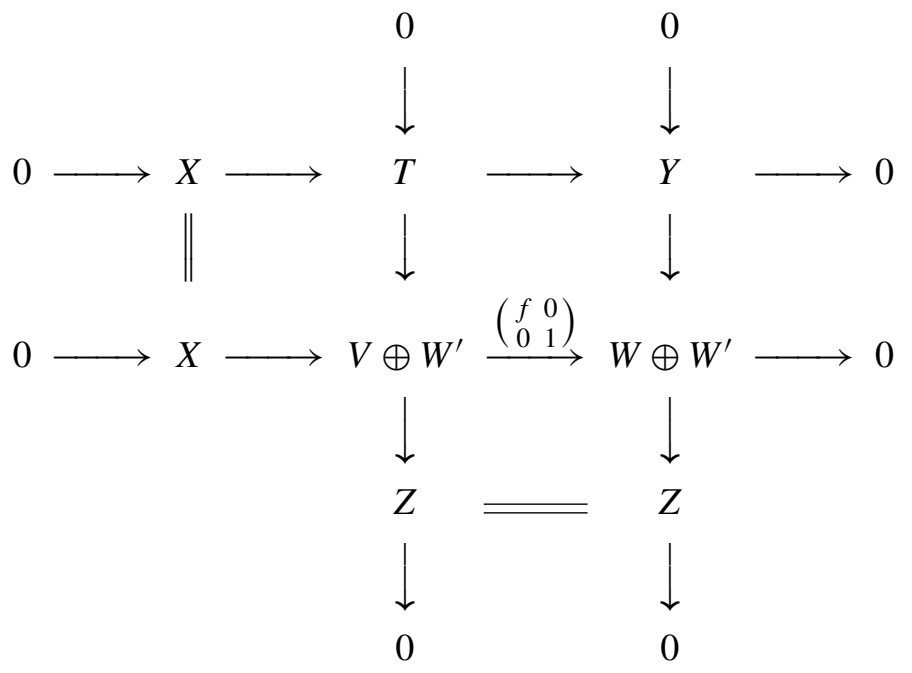

We see from the first row that $T$ is in $\mathscr{X} \bullet \mathscr{Y}$, and from the middle column that $M$ is in $(\mathscr{X} \bullet \mathcal{Y}) \bullet \mathscr{L}$. Therefore $\mathscr{X} \bullet(\mathscr{Y} \bullet \mathscr{L}) \subseteq(\mathscr{X} \bullet \mathscr{Y}) \bullet \mathscr{L}$ holds.

(3) It is enough to show the equality $[\mathscr{C}]_{a} \bullet\left[\mathfrak{C}_{b}=[\mathfrak{C}]_{a+b}\right.$. We prove this by induction on $b$. It holds by definition when $b=1$. Let $b \geq 2$. Then we have $[\mathfrak{C}]_{a} \bullet[\mathfrak{C}]_{b}=[\mathscr{C}]_{a} \bullet\left([\mathscr{C}]_{b-1} \bullet \mathscr{C}\right)=\left([\mathfrak{C}]_{a} \bullet[\mathfrak{C}]_{b-1}\right) \bullet \mathscr{C}=[\mathfrak{C}]_{a+b-1} \bullet \mathscr{C}=[\mathfrak{C}]_{a+b}$, where the second equality follows from (2), and the induction hypothesis implies the third equality.

Let $\mathscr{C}$ be a subcategory of $\bmod R$ and $r \geq 0$ an integer. By the second and third assertions of Proposition 2.2, without danger of confusion we can write

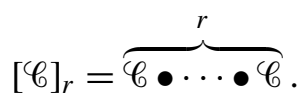

Now we can make the definition of the radius of a subcategory.

Definition 2.3. Let $R$ be a local ring, and let $\mathscr{X}$ be a subcategory of $\bmod R$. We define the radius of $\mathscr{X}$, denoted by radius $\mathscr{X}$, as the infimum of the integers $n \geq 0$ such that there exists a ball of radius $n+1$ centered at a module containing $\mathscr{X}$. By definition, radius $\mathscr{X} \in \mathbb{N} \cup\{\infty\}$. 
The definition of the radius of a resolving subcategory looks similar to that of the dimension of a triangulated category which has been introduced by Rouquier [2008, Definition 3.2]. The stable category $\underline{\mathrm{CM}}(R)$ of Cohen-Macaulay modules over a Gorenstein local ring $R$ is triangulated by [Buchweitz 1986; Happel 1988], and the dimension of $\underline{\mathrm{CM}}(R)$ in the sense of Rouquier is defined. It might look the same as the radius of $\mathrm{CM}(R)$ in our sense.

However there are (at least) two differences in the definitions:

(1) A defining object for $\operatorname{dim} \underline{\mathrm{CM}}(R)$ is required to be inside the category $\underline{\mathrm{CM}}(R)$, but a defining object for radius $\mathrm{CM}(R)$ is not, i.e., it is enough to be an object of mod $R$. More precisely, $\operatorname{dim} \underline{\mathrm{CM}}(R)$ (respectively, radius $\mathrm{CM}(R))$ is defined as the infimum of the integers $n \geq 0$ such that $\underline{\mathrm{CM}}(R)=\langle G\rangle_{n+1}$ for some object $G$ (respectively, $\mathrm{CM}(R) \subseteq[C]_{n+1}$ for some object $C$ ). Then $G$ must be an object of $\underline{\mathrm{CM}}(R)$, while $C$ may not be an object of $\mathrm{CM}(R)$, just being an object of $\bmod R$.

(2) Let $\mathscr{X}$ and $\mathscr{Y}$ be subcategories of $\operatorname{CM}(R)$ and $\underline{\operatorname{CM}}(R)$, respectively. Then the subcategory $\langle y\rangle$ of $\underline{\mathrm{CM}}(R)$ is closed under taking cosyzygies of CohenMacaulay modules in it, but the subcategory [Ø] of $\operatorname{CM}(R)$ is not in general. (In fact, this difference is a reason why we can prove Proposition 2.5 below but do not know whether the analogue for dimension holds or not; see Question 2.6 below.)

Thus these two notions are different, but they are still related to each other. In fact, we can show that the following relationship exists between them.

Proposition 2.4. Let $R$ be a Gorenstein local ring.

(1) One has the inequality $\operatorname{dim} \underline{\mathrm{CM}}(R) \leq$ radius $\mathrm{CM}(R)$.

(2) The equality holds if $R$ is a hypersurface.

Proof. (1) We may assume that $n:=$ radius $\mathrm{CM}(R)<\infty$. Then there exists an $R$-module $C$ such that $\mathrm{CM}(R)$ is contained in the ball $[C]_{n+1}$.

We claim that $\operatorname{CM}(R)=\left[\Omega^{-d} \Omega^{d} C\right]_{n+1}$ holds, where $d=\operatorname{dim} R$. This claim implies $\underline{\mathrm{CM}}(R)=\left\langle\Omega^{d} C\right\rangle_{n+1}$, which shows $\operatorname{dim} \underline{\mathrm{CM}}(R) \leq n$.

In the following, we show this claim. Since $\Omega^{-d} \Omega^{d} C$ is a Cohen-Macaulay $R$-module, and $\operatorname{CM}(R)$ is a resolving subcategory of $\bmod R$, the inclusion $\operatorname{CM}(R) \supseteq$ $\left[\Omega^{-d} \Omega^{d} C\right]_{n+1}$ holds. To get the opposite inclusion, it is enough to prove that for every $m \geq 1$ and $M \in[C]_{m}$ we have $\Omega^{-d} \Omega^{d} M \in\left[\Omega^{-d} \Omega^{d} C\right]_{m}$. Let us prove this by induction on $m$. The case $m=1$ is obvious, so let $m \geq 2$. According to Proposition 2.2(1), there is an exact sequence $0 \rightarrow X \rightarrow Y \rightarrow Z \rightarrow 0$ of $R$-modules with $X \in[C]_{m-1}$ and $Z \in[C]$ such that $M$ is a direct summand of $Y$. Taking the $d$-th syzygies, we have an exact sequence $0 \rightarrow \Omega^{d} X \rightarrow \Omega^{d} Y \oplus R^{\oplus l} \rightarrow \Omega^{d} Z \rightarrow 0$ 
of Cohen-Macaulay $R$-modules. Since $R$ is Gorenstein, taking the $d$-th cosyzygies makes an exact sequence

$$
0 \rightarrow \Omega^{-d} \Omega^{d} X \rightarrow \Omega^{-d} \Omega^{d} Y \oplus R^{\oplus k} \rightarrow \Omega^{-d} \Omega^{d} Z \rightarrow 0
$$

of Cohen-Macaulay modules. The induction hypothesis implies $\Omega^{-d} \Omega^{d} Z \in$ $\left[\Omega^{-d} \Omega^{d} C\right]$ and $\Omega^{-d} \Omega^{d} X \in\left[\Omega^{-d} \Omega^{d} C\right]_{m-1}$. Since $\Omega^{-d} \Omega^{d} M$ is a direct summand of $\Omega^{-d} \Omega^{d} Y$, it belongs to $\left[\Omega^{-d} \Omega^{d} C\right]_{m}$.

(2) Let $n:=\operatorname{dim} \underline{\operatorname{CM}}(R)<\infty$. We find a Cohen-Macaulay $R$-module $G$ such that $\underline{\mathrm{CM}}(R)=\langle G\rangle_{n+1}$. We want to prove that $\mathrm{CM}(R)=[G]_{n+1}$ holds, and it suffices to show that for every $m \geq 1$ and $M \in\langle G\rangle_{m}$ we have $M \in[G]_{m}$. Let us use induction on $m$. The case $m=1$ follows from the fact that $\Omega N \cong \Omega^{-1} N$ up to free summand for each Cohen-Macaulay $R$-module $N$, since $R$ is a hypersurface. When $m \geq 2$, there exists an exact triangle $X \rightarrow Y \rightarrow Z \rightarrow \Sigma X$ in $\underline{\mathrm{CM}}(R)$ with $X \in\langle G\rangle_{m-1}$ and $Z \in\langle G\rangle$ such that $M$ is a direct summand of $Y$. Then we have an exact sequence $0 \rightarrow X \rightarrow Y \oplus R^{\oplus h} \rightarrow Z \rightarrow 0$ of $R$-modules, and we are done by applying the induction hypothesis.

In the rest of this section, we will study the relationships between the representation types of a Cohen-Macaulay local ring and the radius of the category of Cohen-Macaulay modules. Recall that a Cohen-Macaulay local ring $R$ is said to be of finite (respectively, countable) Cohen-Macaulay representation type if $\operatorname{CM}(R)$ has only finitely (respectively, countably but not finitely) many indecomposable modules up to isomorphism.

We can describe the property of finite Cohen-Macaulay representation type in terms of a radius.

Proposition 2.5. Let $R$ be a Gorenstein Henselian local ring. The following are equivalent:

(1) One has radius $\mathrm{CM}(R)=0$;

(2) The ring $R$ has finite Cohen-Macaulay representation type.

Proof. (2) $\Rightarrow$ (1): If $M_{1}, \ldots, M_{r}$ are the nonisomorphic indecomposable CohenMacaulay $R$-modules, then we have $\operatorname{CM}(R)=\left[M_{1} \oplus \cdots \oplus M_{r}\right]$.

$(1) \Rightarrow(2)$ : There is an $R$-module $C$ satisfying $\operatorname{CM}(R) \subseteq[C]$. Setting $d=\operatorname{dim} R$, we have $\operatorname{CM}(R)=\left[\Omega^{-d} \Omega^{d} C\right]$. Replacing $C$ with $\Omega^{-d} \Omega^{d} C$, we may assume that $\mathrm{CM}(R)=[C]$ with $C$ being Cohen-Macaulay.

Note that since $R$ is Henselian, the Krull-Schmidt theorem holds, i.e., each $R$-module uniquely decomposes into indecomposable $R$-modules up to isomorphism. Let $C_{1}, \ldots, C_{n}$ be the indecomposable direct summands of $C$. We may assume that $C=C_{1} \oplus \cdots \oplus C_{n}$. Since $R$ is Gorenstein, taking syzygies preserves 
indecomposability of nonfree Cohen-Macaulay $R$-modules. We see that the set of nonisomorphic indecomposable Cohen-Macaulay $R$-modules is

$$
\{R\} \cup\left\{\Omega^{i} C_{j} \mid i \geq 0,1 \leq j \leq n\right\} .
$$

We may assume that for all $i \geq 0$ and $1 \leq j \neq j^{\prime} \leq n$ we have $\Omega^{i} C_{j} ¥ C_{j^{\prime}}$, because if $\Omega^{i} C_{j} \cong C_{j^{\prime}}$ for some such $i, j, j^{\prime}$, then we can exclude $C_{j^{\prime}}$ from $C$. Now fix an integer $j$ with $1 \leq j \leq n$. As taking cosyzygies preserves indecomposability of nonfree Cohen-Macaulay $R$-modules, $\Omega^{-1} C_{j}$ is isomorphic to $\Omega^{a} C_{b}$ for some $a \geq 0$ and $1 \leq b \leq n$. Taking the $a$-th cosyzygies, we have $\Omega^{-1-a} C_{j} \cong C_{b}$, hence $C_{j} \cong \Omega^{1+a} C_{b}$. This forces us to have $b=j$, which says that $C_{j}$ is periodic. Hence there are only finitely many indecomposable Cohen-Macaulay $R$-modules.

Question 2.6. Does the equality in Proposition 2.4(1) hold true? If it does, then Proposition 2.5 will say that a Gorenstein Henselian local ring $R$ has finite CohenMacaulay representation type if and only if $\operatorname{dim} \mathrm{CM}(R) \leq 0$. This statement is a partial generalization of Minamoto's theorem [2013, Theorem 0.2], which asserts that the same statement holds for a finite-dimensional self-injective algebra over a perfect field, extending Yoshiwaki's recent theorem [2011, Corollary 3.10].

The next result hints at further relationship between finite radius of $\operatorname{CM}(R)$ and more well-known classification of singularities.

Proposition 2.7. Let $R$ be a complete local hypersurface over an algebraically closed field of characteristic not two. Assume that $R$ is of countable CohenMacaulay representation type. Then radius $\mathrm{CM}(R)=1$.

Proof. It follows from [Araya et al. 2012, Theorem 1.1] that there exists an $R$-module $X$ such that for every indecomposable module $M \in \mathrm{CM}(R)$ there is an exact sequence $0 \rightarrow L \rightarrow M \oplus R^{n} \rightarrow N \rightarrow 0$ with $L, N \in\{0, X, \Omega X\}$. This shows that $\mathrm{CM}(R)=[X]_{2}$. Now we see from Proposition 2.5 that the radius of $\mathrm{CM}(R)$ is equal to one.

\section{Finiteness of the radius of a resolving subcategory}

In this section we state our guiding conjecture and first main result.

Conjecture 3.1. Let $R$ be a Cohen-Macaulay local ring. Let $\mathscr{X}$ be a resolving subcategory of $\bmod R$ with finite radius. Then every $R$-module in $\mathscr{X}$ is CohenMacaulay.

Remark 3.2. The converse of Conjecture 3.1 also seems to be true. We consider this in Section 5.

Let $\mathscr{X}$ be a subcategory of $\bmod R$. We denote by res $\mathscr{X}$ (or $\operatorname{res}_{R} \mathscr{X}$ ) the resolving closure of $\mathscr{X}$, namely, the smallest resolving subcategory of $\bmod R$ containing $\mathscr{X}$. If 
$\mathscr{X}$ consists of a single module $M$, then we simply denote it by res $M$ (or $\operatorname{res}_{R} M$ ). For a prime ideal $\mathfrak{p}$ of $R$, we denote by $\mathscr{X}_{\mathfrak{p}}$ the subcategory of mod $R_{\mathfrak{p}}$ consisting of all modules of the form $X_{\mathfrak{p}}$, where $X \in \mathscr{X}$. The first main result of this paper is the following theorem.

Theorem 3.3. Let $R$ be a commutative Noetherian ring. Let $\mathscr{X}$ be a resolving subcategory of $\bmod R$. Suppose that there exist a prime ideal $\mathfrak{p}$ of $R$ with $\mathrm{ht} \mathfrak{p}>0$ and an $R_{\mathfrak{p}}$-module $M$ with $0 \neq M \in \operatorname{add}_{R_{\mathfrak{p}}} \mathscr{X}_{\mathfrak{p}}$ which satisfy one of the following conditions.

(1) $\mathfrak{p} M=0$.

(2) $0<\operatorname{Gdim}_{R_{\mathfrak{p}}} M=n<\infty$ and $\Omega_{R_{\mathfrak{p}}}^{-2} \Omega_{R_{\mathfrak{p}}}^{n} M \in \operatorname{add}_{R_{\mathfrak{p}}} \mathscr{X}_{\mathfrak{p}}$.

(3) $0<\operatorname{Gdim}_{R_{\mathfrak{p}}} M<\infty$ and $\operatorname{res}_{R_{\mathfrak{p}}}\left(\Omega_{R_{\mathfrak{p}}}^{n} M\right)$ is a thick subcategory of $\mathscr{G}\left(R_{\mathfrak{p}}\right)$ for some $n \geq 0$.

(4) $0<\operatorname{Cldim}_{R_{\mathfrak{p}}} M<\infty$.

Then $\mathscr{Q}$ has infinite radius.

The proof of this theorem will be given in the next section. As a direct consequence of the above theorem, we obtain two cases in which our conjecture holds true.

Corollary 3.4. Conjecture 3.1 is true if

(1) $R$ is a complete intersection, or

(2) $R$ is Gorenstein, and every resolving subcategory of $\bmod R$ contained in $\mathrm{CM}(R)$ is a thick subcategory of $\mathrm{CM}(R)$.

Proof. Conjecture 3.1 trivially holds in the case where $R$ is $\operatorname{Artinian,~so~let~}(R, \mathfrak{m}, k)$ be a Cohen-Macaulay local ring of positive dimension. Then we have ht $\mathfrak{m}>0$. Let $\mathscr{L}$ be a resolving subcategory of $\bmod R$, and suppose that $\mathscr{X}$ contains a nonCohen-Macaulay $R$-module $M$.

(1) We have $0<\operatorname{dim} R-\operatorname{depth}_{R} M=\operatorname{depth} R-\operatorname{depth}_{R} M=\operatorname{Cldim}_{R} M<\infty$, and Theorem 3.3(4) implies that $\mathscr{X}$ has infinite radius.

(2) We have $0<n:=\operatorname{dim} R-\operatorname{depth}_{R} M=\operatorname{depth} R-\operatorname{depth}_{R} M=\operatorname{Gdim}_{R} M<\infty$. The module $\Omega_{R}^{n} M$ is Cohen-Macaulay, and by assumption $\operatorname{res}_{R}\left(\Omega_{R}^{n} M\right)$ is a thick subcategory of $\mathrm{CM}(R)=\mathscr{G}(R)$. Theorem 3.3(3) implies that $\mathscr{L}$ has infinite radius.

\section{Proof of Theorem I}

This section is devoted to give the proof of Theorem 3.3 (hence of Theorem I from the introduction), which we break up into several parts. Most of them also reveal properties of subcategories of $\bmod R$ which are interesting in their own right.

First of all, we make a remark to reduce our theorem to the local case. 
Remark 4.1. Let $C$ be an $R$-module, $n \geq 0$ an integer and $\mathfrak{p}$ a prime ideal of $R$. Then for a subcategory $\mathscr{X}$ of $\bmod R$ the implication

$$
\mathscr{L} \subseteq[C]_{n}^{R} \Rightarrow \operatorname{add}_{R_{\mathfrak{p}}} \mathscr{X}_{\mathfrak{p}} \subseteq\left[C_{\mathfrak{p}}\right]_{n}^{R_{\mathfrak{p}}}
$$

holds. It follows from [Takahashi 2010, Lemma 4.8] that $\operatorname{add}_{R_{\mathfrak{p}}} \mathscr{X}_{\mathfrak{p}}$ is a resolving subcategory of $\bmod R_{\mathfrak{p}}$. (The ring $R$ in (loc. cit.) is assumed to be local, but its proof does not use this assumption, so it holds for an arbitrary commutative Noetherian ring.) Hence, to prove Theorem 3.3, without loss of generality we can assume $(R, \mathfrak{p})$ is a local ring with $\operatorname{dim} R>0$ and $M$ is an $R$-module with $0 \neq M \in \mathscr{X}$.

Proof of Theorem 3.3(1). First, we investigate the annihilators of torsion submodules. For an ideal $I$ of $R$ and an $R$-module $M$, we denote by $\Gamma_{I}(M)$ the $I$-torsion submodule of $M$. Recall that $\Gamma_{I}(M)$ is by definition the subset of $M$ consisting of all elements that are annihilated by some power of $I$, and the assignment $M \mapsto \Gamma_{I}(M)$ defines a left exact additive covariant functor $\Gamma_{I}: \bmod R \rightarrow \bmod R$.

Lemma 4.2. Let $I$ be an ideal of $R$. Let $C, M$ be $R$-modules and $n \geq 1$ an integer. If $M$ belongs to $[C]_{n}$, then one has $\operatorname{Ann}_{R} \Gamma_{I}(M) \supseteq\left(\operatorname{Ann}_{R} \Gamma_{I}(R) \cdot \operatorname{Ann}_{R} \Gamma_{I}(C)\right)^{n}$.

Proof. Let us prove the lemma by induction on $n$.

If $n=1$, the module $M$ is isomorphic to a direct summand of $\left(R \oplus \bigoplus_{i=0}^{a} \Omega^{i} C\right)^{\oplus b}$ for some $a, b \geq 0$. Hence $\Gamma_{I}(M)$ is isomorphic to a direct summand of

$$
\left(\Gamma_{I}(R) \oplus \bigoplus_{i=0}^{a} \Gamma_{I}\left(\Omega^{i} C\right)\right)^{\oplus b} .
$$

For $i \geq 1$, the syzygy $\Omega^{i} C$ is a submodule of some free module $R^{\oplus c_{i}}$, and $\Gamma_{I}\left(\Omega^{i} C\right)$ is a submodule of $\Gamma_{I}(R)^{\oplus c_{i}}$, which implies that $\operatorname{Ann}_{R} \Gamma_{I}\left(\Omega^{i} C\right)$ contains $\operatorname{Ann}_{R} \Gamma_{I}(R)$. Hence we obtain

$$
\begin{aligned}
\operatorname{Ann}_{R} \Gamma_{I}(M) & \supseteq \operatorname{Ann}_{R} \Gamma_{I}(R) \cap\left(\bigcap_{i=0}^{a} \operatorname{Ann}_{R} \Gamma_{I}\left(\Omega^{i} C\right)\right) \\
& =\operatorname{Ann}_{R} \Gamma_{I}(R) \cap \operatorname{Ann}_{R} \Gamma_{I}(C) \supseteq \operatorname{Ann}_{R} \Gamma_{I}(R) \cdot \operatorname{Ann}_{R} \Gamma_{I}(C) .
\end{aligned}
$$

Let $n \geq 2$. Then $M$ is in $[C]_{n}=[C]_{n-1} \bullet[C]$, and Proposition 2.2(1) says that there is an exact sequence $0 \rightarrow X \rightarrow Y \rightarrow Z \rightarrow 0$ with $X \in[C]_{n-1}$ and $Z \in[C]$ such that $M$ is a direct summand of $Y$. We have an exact sequence $0 \rightarrow \Gamma_{I}(X) \rightarrow \Gamma_{I}(Y) \rightarrow \Gamma_{I}(Z)$, and therefore we obtain

$$
\begin{aligned}
\operatorname{Ann}_{R} \Gamma_{I}(M) & \supseteq \operatorname{Ann}_{R} \Gamma_{I}(Y) \supseteq \operatorname{Ann}_{R} \Gamma_{I}(X) \cdot \operatorname{Ann}_{R} \Gamma_{I}(Z) \\
& \supseteq\left(\operatorname{Ann}_{R} \Gamma_{I}(R) \cdot \operatorname{Ann}_{R} \Gamma_{I}(C)\right)^{n-1} \cdot\left(\operatorname{Ann}_{R} \Gamma_{I}(R) \cdot \operatorname{Ann}_{R} \Gamma_{I}(C)\right) \\
& =\left(\operatorname{Ann}_{R} \Gamma_{I}(R) \cdot \operatorname{Ann}_{R} \Gamma_{I}(C)\right)^{n}
\end{aligned}
$$


which is what we want.

Let $(R, \mathfrak{m})$ be a local ring, and let $M$ be an $R$-module. We denote by $\ell \ell(M)$ the Loewy length of $M$, which is by definition the infimum of the integers $n \geq 0$ such that $\mathfrak{m}^{n} M=0$. Obviously, $\ell \ell(M)$ is finite if and only if $M$ has finite length. There is a relationship between finite radius and Loewy length:

Proposition 4.3. Let $(R, \mathfrak{m})$ be a local ring, and let $\mathscr{L}$ be a resolving subcategory of $\bmod R$. If radius $\mathscr{X}<\infty$, then $\sup _{X \in \mathscr{X}}\left\{\ell \ell\left(\Gamma_{\mathfrak{m}}(X)\right)\right\}<\infty$.

Proof. Put $r=$ radius $\mathscr{X}$. By definition, there exists an $R$-module $C$ such that $[C]_{r+1}$ contains $\mathscr{X}$. Let $X$ be a module in $\mathscr{X}$. It follows from Lemma 4.2 that the annihilator Ann $\Gamma_{\mathfrak{m}}(X)$ contains the ideal $\left(\operatorname{Ann} \Gamma_{\mathfrak{m}}(R) \cdot \operatorname{Ann} \Gamma_{\mathfrak{m}}(C)\right)^{r+1}$. As $\Gamma_{\mathfrak{m}}(R)$ and $\Gamma_{\mathfrak{m}}(C)$ have finite length, they have finite Loewy length. Set $a=\ell \ell\left(\Gamma_{\mathfrak{m}}(R)\right)$ and $b=\ell \ell\left(\Gamma_{\mathfrak{m}}(C)\right)$. Then Ann $\Gamma_{\mathfrak{m}}(X)$ contains $\mathfrak{m}^{(a+b)(r+1)}$, which means that $\ell \ell\left(\Gamma_{\mathfrak{m}}(X)\right)$ is at most $(a+b)(r+1)$. Since the number $(a+b)(r+1)$ is independent of the choice of $X$, we have $\sup _{X \in \mathscr{L}}\left\{\ell \ell\left(\Gamma_{\mathfrak{m}}(X)\right)\right\} \leq(a+b)(r+1)<\infty$.

The following is the essential part of Theorem 3.3(1).

Theorem 4.4. Let $(R, \mathfrak{m}, k)$ be a local ring of positive dimension. Let $\mathscr{X}$ be a resolving subcategory of $\bmod R$. If $\mathscr{X}$ contains $k$, then $\mathscr{X}$ has infinite radius.

Proof. We claim that $R / \mathfrak{m}^{i}$ belongs to $\mathscr{X}$ for all integers $i>0$. Indeed, there is an exact sequence $0 \rightarrow \mathfrak{m}^{i-1} / \mathfrak{m}^{i} \rightarrow R / \mathfrak{m}^{i} \rightarrow R / \mathfrak{m}^{i-1} \rightarrow 0$, and the left term belongs to $\mathscr{L}$ as it is a $k$-vector space. Induction on $i$ shows the claim.

We have $\ell \ell\left(\Gamma_{\mathfrak{m}}\left(R / \mathfrak{m}^{i}\right)\right)=\ell \ell\left(R / \mathfrak{m}^{i}\right)=i$, where the second equality follows from the assumption that $\operatorname{dim} R>0$. Therefore it holds that

$$
\sup _{X \in \mathscr{R}}\left\{\ell \ell\left(\Gamma_{\mathfrak{m}}(X)\right)\right\} \geq \sup _{i>0}\left\{\ell \ell\left(\Gamma_{\mathfrak{m}}\left(R / \mathfrak{m}^{i}\right)\right)\right\}=\sup _{i>0}\{i\}=\infty,
$$

which implies radius $\mathscr{X}=\infty$ by Proposition 4.3.

Now, let us prove Theorem 3.3(1). By Remark 4.1, we may assume that $(R, \mathfrak{p})$ is a local ring with $\operatorname{dim} R>0$ and that $M$ is a nonzero $R$-module in $\mathscr{X}$. The assumption $\mathfrak{p} M=0$ says that $M$ is a nonzero $k$-vector space, where $k=R / \mathfrak{p}$ is the residue field of $R$. Since $\mathscr{X}$ is closed under direct summands, $k$ belongs to $\mathscr{X}$. Theorem 4.4 yields radius $\mathscr{X}=\infty$.

Proof of Theorem 3.3(2)(3). Establishing several preliminary lemmas and propositions is necessary, which will also be used in the proof of Theorem 3.3(4).

We begin with stating an elementary lemma, whose proof we omit.

Lemma 4.5. Let $(R, \mathfrak{m}, k)$ be a local ring. Let $0 \rightarrow L \rightarrow M \rightarrow N \rightarrow 0$ be an exact sequence of $R$-modules. Then $\inf \{\operatorname{depth} L$, depth $N\}=\inf \{\operatorname{depth} M$, depth $N\}$. 
For an ideal $I$ of $R$, we denote by $V(I)$ (respectively, $D(I)$ ) the closed (respectively, open) subset of Spec $R$ defined by $I$ in the Zariski topology, namely, $V(I)$ is the set of prime ideals containing $I$ and $D(I)=\operatorname{Spec} R \backslash V(I)$. For an $R$-module $M$ we denote by $\mathrm{NF}(M)$ the nonfree locus of $M$, that is, the set of prime ideals $\mathfrak{p}$ of $R$ such that the $R_{\mathfrak{p}}$-module $M_{\mathfrak{p}}$ is nonfree. It is well-known that $\mathrm{NF}(M)$ is a closed subset of $\operatorname{Spec} R$.

The next result builds, out of each module in a resolving subcategory and each point in its nonfree locus, another module in the same resolving subcategory whose nonfree locus coincides with the closure of the point. Such a construction has already been given in [Takahashi 2009, Theorem 4.3], but we need in this paper a more detailed version. Indeed, the following lemma yields a generalization of (loc. cit.).

Lemma 4.6. Let $M$ be an $R$-module. For every $\mathfrak{p} \in \mathrm{NF}(M)$ there exists $X \in \operatorname{res} M$ satisfying $\operatorname{NF}(X)=V(\mathfrak{p})$ and depth $X_{\mathfrak{q}}=\inf \left\{\right.$ depth $M_{\mathfrak{q}}$, depth $\left.R_{\mathfrak{q}}\right\}$ for all $\mathfrak{q} \in V(\mathfrak{p})$.

Proof. Note that $V(\mathfrak{p})$ is contained in $\mathrm{NF}(M)$. If $V(\mathfrak{p})=\mathrm{NF}(M)$, then we can take $X:=M \oplus R$. Suppose $V(\mathfrak{p})$ is strictly contained in $\operatorname{NF}(M)$. Then there is a prime ideal $\mathfrak{r}$ in $\operatorname{NF}(M)$ that is not in $V(\mathfrak{p})$. Choose an element $x \in \mathfrak{p} \backslash \mathfrak{r}$. By [ibid., Proposition 4.2], we have a commutative diagram with exact rows

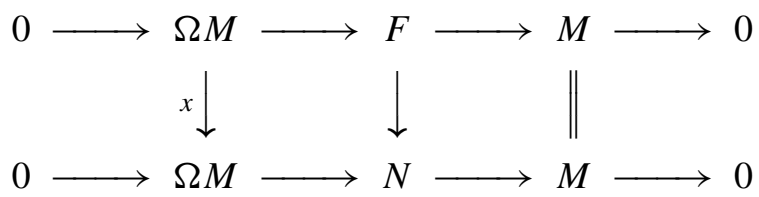

where $F$ is free, $V(\mathfrak{p}) \subseteq \mathrm{NF}(N) \subseteq \mathrm{NF}(M)$ and $D((x)) \cap \mathrm{NF}(N)=\varnothing$. The second row shows that $N$ belongs to res $M$. Since $\mathfrak{r}$ is in $D((x))$, it is not in $\operatorname{NF}(N)$, and we have $V(\mathfrak{p}) \subseteq \mathrm{NF}(N) \subsetneq \mathrm{NF}(M)$.

Now we claim that depth $N_{\mathfrak{q}}=\inf \left\{\right.$ depth $M_{\mathfrak{q}}$, depth $\left.R_{\mathfrak{q}}\right\}$ for all $\mathfrak{q} \in V(\mathfrak{p})$. Indeed, localizing the above diagram at $\mathfrak{q}$ and taking long exact sequences with respect to Ext, we get a commutative diagram with exact rows

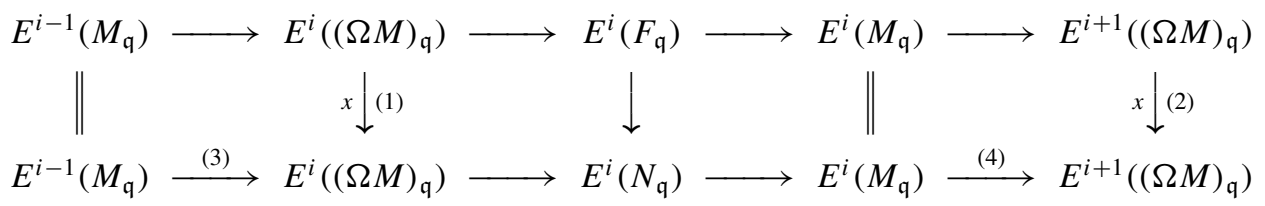

for $i \in \mathbb{Z}$, where $E^{i}(-)=\operatorname{Ext}_{R_{\mathfrak{q}}}^{i}(\kappa(\mathfrak{q}),-)$. As $x$ is an element of $\mathfrak{q}$, the maps (1), (2) are zero maps, and so are (3), (4). Thus we have a short exact sequence

$$
0 \rightarrow \operatorname{Ext}^{i}\left(\kappa(\mathfrak{q}),(\Omega M)_{\mathfrak{q}}\right) \rightarrow \operatorname{Ext}^{i}\left(\kappa(\mathfrak{q}), N_{\mathfrak{q}}\right) \rightarrow \operatorname{Ext}^{i}\left(\kappa(\mathfrak{q}), M_{\mathfrak{q}}\right) \rightarrow 0
$$

for each integer $i$. It is easy to see from this that the first equality in the following holds, while the second equality is obtained by applying Lemma 4.5 to the exact 
sequence $0 \rightarrow(\Omega M)_{\mathfrak{q}} \rightarrow F_{\mathfrak{q}} \rightarrow M_{\mathfrak{q}} \rightarrow 0$ :

$\operatorname{depth} N_{\mathfrak{q}}=\inf \left\{\operatorname{depth}(\Omega M)_{\mathfrak{q}}, \operatorname{depth} M_{\mathfrak{q}}\right\}=\inf \left\{\operatorname{depth} R_{\mathfrak{q}}, \operatorname{depth} M_{\mathfrak{q}}\right\}$.

Thus the claim follows.

If $V(\mathfrak{p})=\operatorname{NF}(N)$, then we can take $X:=N$. If $V(\mathfrak{p})$ is strictly contained in $\mathrm{NF}(N)$, then the above procedure gives rise to an $R$-module $L \in \operatorname{res} N$ (so $L \in \operatorname{res} M$ ) with $V(\mathfrak{p}) \subseteq \mathrm{NF}(L) \subsetneq \mathrm{NF}(N) \subsetneq \mathrm{NF}(M)$ such that

$\operatorname{depth} L_{\mathfrak{q}}=\inf \left\{\operatorname{depth} N_{\mathfrak{q}}\right.$, depth $\left.R_{\mathfrak{q}}\right\}=\inf \left\{\operatorname{depth} M_{\mathfrak{q}}, \operatorname{depth} R_{\mathfrak{q}}\right\}$

for all $\mathfrak{q} \in V(\mathfrak{p})$. Since Spec $R$ is a Noetherian space, iteration of this procedure must stop in finitely many steps, and we eventually obtain such a module $X$ as in the lemma.

The next lemma will play a crucial role in the proofs of our theorems. The main idea of the proof is similar to that of Lemma 4.2, but a much closer examination is necessary.

Lemma 4.7. Let $S \rightarrow R$ be a homomorphism of rings. Let $C, M$ be $R$-modules with $M \in[C]_{n}^{R}$, and let $N$ be an $S$-module of injective dimension $m<\infty$. Then

$$
\begin{aligned}
\bigcap_{i>0} \operatorname{Ann}_{S} \operatorname{Ext}_{S}^{i}(M, N) & =\bigcap_{i=1}^{m} \operatorname{Ann}_{S} \operatorname{Ext}_{S}^{i}(M, N) \\
& \supseteq\left(\prod_{i=1}^{m} \operatorname{Ann}_{S} \operatorname{Ext}_{S}^{i}(R, N) \cdot \operatorname{Ann}_{S} \operatorname{Ext}_{S}^{i}(C, N)\right)^{n} .
\end{aligned}
$$

Proof. For each integer $i \leq 1$ and $R$-module $L$, set $\mathfrak{a}_{L}^{i}=\operatorname{Ann}_{S} \operatorname{Ext}_{S}^{i}(L, N)$. Note that $\mathfrak{a}_{L}^{h}=S$ for all $h>m$ since $\operatorname{Ext}_{S}^{h}(L, N)=0$. It suffices to prove that $\mathfrak{a}_{M}^{i} \supseteq\left(\prod_{j=i}^{m} \mathfrak{a}_{R}^{j} \mathfrak{a}_{C}^{j}\right)^{n}$. Let us proceed by induction on $n$.

When $n=0$, we have $M=0$, and the above two ideals coincide with $S$.

Let $n=1$. Then $M$ is isomorphic to a direct summand of a finite direct sum of copies of $R \oplus\left(\oplus_{j=0}^{l} \Omega^{j} C\right)$. Hence $\operatorname{Ext}_{S}^{i}(M, N)$ is isomorphic to a direct summand of a finite direct sum of copies of $\operatorname{Ext}_{S}^{i}(R, N) \oplus\left(\bigoplus_{j=0}^{l} \operatorname{Ext}_{S}^{i}\left(\Omega^{j} C, N\right)\right)$. Thus we have $\mathfrak{a}_{M}^{i} \supseteq \mathfrak{a}_{R}^{i} \cap\left(\bigcap_{j=0}^{l} \mathfrak{a}_{\Omega^{j} C}^{i}\right)$. For each $j \geq 1$ there is an exact sequence $0 \rightarrow \Omega^{j} C \rightarrow R^{\oplus k_{j}} \rightarrow \Omega^{j-1} C \rightarrow 0$, which induces an exact sequence

$$
\operatorname{Ext}_{S}^{i}(R, N)^{\oplus k_{j}} \rightarrow \operatorname{Ext}_{S}^{i}\left(\Omega^{j} C, N\right) \rightarrow \operatorname{Ext}_{S}^{i+1}\left(\Omega^{j-1} C, N\right) .
$$

This gives

$$
\mathfrak{a}_{\Omega^{j} C}^{i} \supseteq \mathfrak{a}_{R}^{i} \mathfrak{a}_{\Omega^{j-1} C}^{i+1} \supseteq \mathfrak{a}_{R}^{i} \mathfrak{a}_{R}^{i+1} \mathfrak{a}_{\Omega^{j-2} C}^{i+2} \supseteq \cdots \supseteq \mathfrak{a}_{R}^{i} \mathfrak{a}_{R}^{i+1} \cdots \mathfrak{a}_{R}^{i+j-1} \mathfrak{a}_{C}^{i+j} .
$$


Regarding $\mathfrak{a}_{R}^{i} \mathfrak{a}_{R}^{i+1} \cdots \mathfrak{a}_{R}^{i+j-1} \mathfrak{a}_{C}^{i+j}$ as $\mathfrak{a}_{C}^{i}$ when $j=0$, we have

$$
\mathfrak{a}_{\Omega^{j} C}^{i} \supseteq \mathfrak{a}_{R}^{i} \mathfrak{a}_{R}^{i+1} \cdots \mathfrak{a}_{R}^{i+j-1} \mathfrak{a}_{C}^{i+j}
$$

for all $j \geq 0$. Thus we obtain

$$
\begin{aligned}
\mathfrak{a}_{M}^{i} & \supseteq \mathfrak{a}_{R}^{i} \cap\left(\bigcap_{j=0}^{l} \mathfrak{a}_{R}^{i} \mathfrak{a}_{R}^{i+1} \cdots \mathfrak{a}_{R}^{i+j-1} \mathfrak{a}_{C}^{i+j}\right) \\
& \supseteq\left(\mathfrak{a}_{R}^{i} \mathfrak{a}_{R}^{i+1} \cdots \mathfrak{a}_{R}^{m} \mathfrak{a}_{R}^{m+1} \cdots\right)\left(\mathfrak{a}_{C}^{i} \mathfrak{a}_{C}^{i+1} \cdots \mathfrak{a}_{C}^{m} \mathfrak{a}_{C}^{m+1} \cdots\right) \\
& =\left(\mathfrak{a}_{R}^{i} \mathfrak{a}_{R}^{i+1} \cdots \mathfrak{a}_{R}^{m}\right)\left(\mathfrak{a}_{C}^{i} \mathfrak{a}_{C}^{i+1} \cdots \mathfrak{a}_{C}^{m}\right) \supseteq \prod_{j=i}^{m} \mathfrak{a}_{R}^{j} \mathfrak{a}_{C}^{j} .
\end{aligned}
$$

Now let us consider the case where $n \geq 2$. We have $M \in[C]_{n}=[C]_{n-1} \bullet[C]$, and by Proposition 2.2(1), there exists an exact sequence $0 \rightarrow X \rightarrow Y \rightarrow Z \rightarrow 0$ with $X \in[C]_{n-1}$ and $Z \in[C]$ such that $M$ is a direct summand of $Y$. Using the induction hypothesis, we have

$$
\mathfrak{a}_{M}^{i} \supseteq \mathfrak{a}_{Y}^{i} \supseteq \mathfrak{a}_{X}^{i} \cdot \mathfrak{a}_{Z}^{i} \supseteq\left(\prod_{j=i}^{m} \mathfrak{a}_{R}^{j} \mathfrak{a}_{C}^{j}\right)^{n-1} \cdot\left(\prod_{j=i}^{m} \mathfrak{a}_{R}^{j} \mathfrak{a}_{C}^{j}\right)=\left(\prod_{j=i}^{m} \mathfrak{a}_{R}^{j} \mathfrak{a}_{C}^{j}\right)^{n}
$$

which completes the proof of the lemma.

Here we prepare a lemma, which is an easy consequence of Krull's intersection theorem.

Lemma 4.8. Let $(R, \mathfrak{m})$ be a local ring and $M$ an $R$-module. Then $\operatorname{Ann}_{R} M=$ $\bigcap_{i>0} \operatorname{Ann}_{R}\left(M / \mathfrak{m}^{i} M\right)$.

Now we can prove the following proposition, which will be the base of the proofs of our theorems. Actually, all of them will be proved by making use of this proposition.

Proposition 4.9. Let $(R, \mathfrak{m}, k)$ be a local ring. Let $\mathscr{X}$ be a resolving subcategory of $\bmod R$. If $\mathscr{X}$ contains a module $M$ such that $0<\operatorname{pd}_{R} M<\infty$, then radius $\mathscr{X}=\infty$.

Proof. Applying Lemma 4.6 to $\mathfrak{m} \in \mathrm{NF}(M)$, we find a module $X \in$ res $M \subseteq \mathscr{X}$ satisfying $\mathrm{NF}(X)=\{\mathfrak{m}\}$ and depth $X=\inf \{$ depth $M$, depth $R\}$. Since $M$ has finite projective dimension, the depth of $M$ is at most that of $R$. Hence, we have depth $X=$ depth $M$. Note that the subcategory of $\bmod R$ consisting of $R$-modules of finite projective dimension is resolving. Since it contains $M$, it also contains res $M$. This implies that $X$ has finite projective dimension, and we have

$$
\operatorname{pd}_{R} X=\text { depth } R-\operatorname{depth} X=\text { depth } R-\operatorname{depth} M=\operatorname{pd}_{R} M \text {. }
$$


Thus, replacing $M$ with $X$, we may assume that $M$ is locally free on the punctured spectrum of $R$. Taking the $n$-th syzygy of $M$ where $n=\operatorname{pd}_{R} M-1 \geq 0$, we may also assume that the projective dimension of $M$ is equal to 1 .

Now $\operatorname{Ext}_{R}^{1}(M, R)$ is a nonzero $R$-module of finite length, and we can choose a socle element $0 \neq \sigma \in \operatorname{Ext}_{R}^{1}(M, R)$. It can be represented as a short exact sequence

$$
\sigma: 0 \rightarrow R \rightarrow N \rightarrow M \rightarrow 0 \text {. }
$$

The module $N$ belongs to $\mathscr{X}$, is locally free on the punctured spectrum of $R$ and has projective dimension at most 1 . Hence $\operatorname{pd}_{R} N=1$ if and only if $\operatorname{Ext}_{R}^{1}(N, R) \neq 0$. Applying the functor $\operatorname{Hom}_{R}(-, R)$, we get an exact sequence

$$
R \stackrel{f}{\rightarrow} \operatorname{Ext}_{R}^{1}(M, R) \rightarrow \operatorname{Ext}_{R}^{1}(N, R) \rightarrow 0,
$$

where $f$ sends $1 \in R$ to $\sigma \in \operatorname{Ext}_{R}^{1}(M, R)$. Hence we obtain an exact sequence

$$
0 \rightarrow k \rightarrow \operatorname{Ext}_{R}^{1}(M, R) \rightarrow \operatorname{Ext}_{R}^{1}(N, R) \rightarrow 0 .
$$

This implies length $\left(\operatorname{Ext}_{R}^{1}(N, R)\right)=$ length $\left(\operatorname{Ext}_{R}^{1}(M, R)\right)-1$. Replacing $M$ by $N$ and repeating this process if length $\left.\operatorname{Ext}_{R}^{1}(N, R)\right)>0$, we can assume that $\operatorname{Ext}_{R}^{1}(N, R)=0$. Therefore $\operatorname{Ext}_{R}^{1}(M, R) \cong k$. Since $\operatorname{pd}_{R} M=1$, we easily get an isomorphism $\operatorname{Tr} M \cong k$. Taking the transpose of this isomorphism, we see that $\operatorname{Tr} k$ is isomorphic to $M$ up to free summand (see [Auslander and Bridger 1969, Proposition (2.6)(d)]). It follows that $\operatorname{Tr} k$ belongs to $\mathscr{X}$.

We claim that $\operatorname{Tr} L$ is in $\mathscr{X}$ for any $R$-module $L$ of finite length. This is shown by induction on length $L$. If length $L>0$, then there is an exact sequence $0 \rightarrow$ $L^{\prime} \rightarrow L \rightarrow k \rightarrow 0$, and applying [ibid., Lemma (3.9)] (see also [Takahashi 2013, Proposition 3.3(3)]), we have an exact sequence

$$
0=\left(L^{\prime}\right)^{*} \rightarrow \operatorname{Tr} k \rightarrow \operatorname{Tr} L \oplus R^{\oplus n} \rightarrow \operatorname{Tr} L^{\prime} \rightarrow 0,
$$

where the equality follows from the fact that $R$ has positive depth. (As $R$ possesses a module of finite positive projective dimension, the depth of $R$ is positive.) The induction hypothesis implies $\operatorname{Tr} L^{\prime} \in \mathscr{X}$, and the above exact sequence shows $\operatorname{Tr} L \in \mathscr{X}$, as desired.

Now, assume that we have radius $\mathscr{X}=r<\infty$. We want to deduce a contradiction.

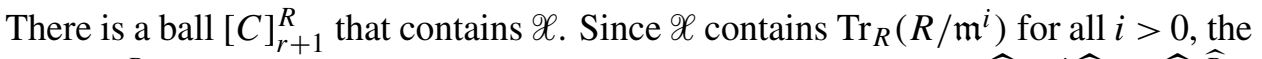
ball $[C]_{r+1}^{R}$ also contains it. Taking the completions, we have $\operatorname{Tr}_{\widehat{R}}\left(\widehat{R} / \mathfrak{m}^{i} \widehat{R}\right) \in[\widehat{C}]_{r+1}^{\widehat{R}}$ for all $i>0$. By virtue of Cohen's structure theorem, there exists a surjective homomorphism $S \rightarrow \widehat{R}$ such that $S$ is a Gorenstein local $\operatorname{ring}$ with $\operatorname{dim} S=\operatorname{dim} R=: d$. Let $\mathfrak{n}$ denote the maximal ideal of $S$ and note that we have $\widehat{R} / \mathfrak{m}^{i} \widehat{R}=\widehat{R} / \mathfrak{n}^{i} \widehat{R}$ for 
any $i>0$. Lemma 4.7 gives an inclusion relation

$$
\bigcap_{j>0} \operatorname{Ann}_{S} \operatorname{Ext}_{S}^{j}\left(\operatorname{Tr}_{\widehat{R}}\left(\widehat{R} / \mathfrak{n}^{i} \widehat{R}\right), S\right) \supseteq\left(\prod_{j=1}^{d} \operatorname{Ann}_{S} \operatorname{Ext}_{S}^{j}(\widehat{R}, S) \cdot \operatorname{Ann}_{S} \operatorname{Ext}_{S}^{j}(\widehat{C}, S)\right)^{r+1} .
$$

Fix an integer $i>0$ and let $a_{1}, \ldots, a_{m}$ be a system of generators of the ideal $\mathfrak{n}^{i}$ of $S$. There is an exact sequence $\widehat{R}^{\oplus m} \stackrel{\left(a_{1}, \ldots, a_{m}\right)}{\longrightarrow} \widehat{R} \rightarrow \widehat{R} / \mathfrak{n}^{i} \widehat{R} \rightarrow 0$ of $\widehat{R}$-modules. Dualizing this by $\widehat{R}$ induces an exact sequence

$$
0=\operatorname{Hom}_{\widehat{R}}\left(\widehat{R} / \mathfrak{n}^{i} \widehat{R}, \widehat{R}\right) \rightarrow \widehat{R} \stackrel{\left(\begin{array}{c}
a_{1} \\
\vdots \\
a_{m}
\end{array}\right)}{\longrightarrow} \widehat{R}^{\oplus m} \rightarrow \operatorname{Tr}_{\widehat{R}}\left(\widehat{R} / \mathfrak{n}^{i} \widehat{R}\right) \rightarrow 0,
$$

where the equality follows since depth $\widehat{R}=\operatorname{depth} R>0$. This makes an exact sequence

$$
\operatorname{Hom}_{S}(\widehat{R}, S)^{\oplus m} \stackrel{\left(a_{1}, \ldots, a_{m}\right)}{\longrightarrow} \operatorname{Hom}_{S}(\widehat{R}, S) \rightarrow \operatorname{Ext}_{S}^{1}\left(\operatorname{Tr}_{\widehat{R}}\left(\widehat{R} / \mathfrak{n}^{i} \widehat{R}\right), S\right),
$$

which yields an injection $\operatorname{Hom}_{S}(\widehat{R}, S) / \mathfrak{n}^{i} \operatorname{Hom}_{S}(\widehat{R}, S) \rightarrow \operatorname{Ext}_{S}^{1}\left(\operatorname{Tr}_{\widehat{R}}\left(\widehat{R} / \mathfrak{n}^{i} \widehat{R}\right), S\right)$. Thus

$$
\begin{aligned}
& \operatorname{Ann}_{S}\left(\operatorname{Hom}_{S}(\widehat{R}, S) / \mathfrak{n}^{i} \operatorname{Hom}_{S}(\widehat{R}, S)\right) \\
& \supseteq \operatorname{Ann}_{S}\left(\operatorname{Ext}_{S}^{1}\left(\operatorname{Tr}_{\widehat{R}}\left(\widehat{R} / \mathfrak{n}^{i} \widehat{R}\right), S\right)\right) \\
& \supseteq \bigcap_{j>0} \operatorname{Ann}_{S} \operatorname{Ext}_{S}^{j}\left(\operatorname{Tr}_{\widehat{R}}\left(\widehat{R} / \mathfrak{n}^{i} \widehat{R}\right), S\right) \supseteq\left(\prod_{j=1}^{d} \operatorname{Ann}_{S} \operatorname{Ext}_{S}^{j}(\widehat{R}, S) \cdot \operatorname{Ann}_{S} \operatorname{Ext}_{S}^{j}(\widehat{C}, S)\right)^{r+1},
\end{aligned}
$$

and we obtain

$$
\begin{aligned}
\left(\prod_{j=1}^{d} \operatorname{Ann}_{S} \operatorname{Ext}_{S}^{j}(\widehat{R}, S) \cdot \operatorname{Ann}_{S} \operatorname{Ext}_{S}^{j}(\widehat{C}, S)\right)^{r+1} & \\
& \subseteq \bigcap_{i>0} \operatorname{Ann}_{S}\left(\operatorname{Hom}_{S}(\widehat{R}, S) / \mathfrak{n}^{i} \operatorname{Hom}_{S}(\widehat{R}, S)\right)=\operatorname{Ann}_{S} \operatorname{Hom}_{S}(\widehat{R}, S),
\end{aligned}
$$

where the equality follows from Lemma 4.8.

Let $I$ be the kernel of the surjection $S \rightarrow \widehat{R}$. Since $\operatorname{dim} S=d=\operatorname{dim} \widehat{R}$, the ideal $I$ of $S$ has height zero. Hence there exists a minimal prime ideal $\mathfrak{p}$ of $S$ which contains $I$. Since we have a ring epimorphism from the Artinian Gorenstein local ring $S_{\mathfrak{p}}$ to $\widehat{R}_{\mathfrak{p}}$, the $\widehat{R}_{\mathfrak{p}}$-module $\operatorname{Hom}_{S}(\widehat{R}, S)_{\mathfrak{p}}=\operatorname{Hom}_{S_{\mathfrak{p}}}\left(\widehat{R}_{\mathfrak{p}}, S_{\mathfrak{p}}\right)$ is isomorphic to the injective hull of the residue field of $\widehat{R}_{\mathfrak{p}}$, which is in particular nonzero. This implies that $\mathfrak{p}$ contains the ideal $\operatorname{Ann}_{S} \operatorname{Hom}_{S}(\widehat{R}, S)$. Therefore, for some integer $1 \leq l \leq d$ the ideal $\mathfrak{p}$ contains either $\operatorname{Ann}_{S} \operatorname{Ext}_{S}^{l}(\widehat{R}, S)$ or $\operatorname{Ann}_{S} \operatorname{Ext}_{S}^{l}(\widehat{C}, S)$. If $\mathfrak{p}$ contains $\operatorname{Ann}_{S} \operatorname{Ext}_{S}^{l}(\widehat{R}, S)$, then we have $\operatorname{Ext}_{S_{\mathfrak{p}}}^{l}\left(\widehat{R}_{\mathfrak{p}}, S_{\mathfrak{p}}\right) \neq 0$, which contradicts the 
fact that $S_{\mathfrak{p}}$ is injective as an $S_{\mathfrak{p}}$-module. Similarly, we have a contradiction when $\mathfrak{p}$ contains $\operatorname{Ann}_{S} \operatorname{Ext}_{S}^{l}(\widehat{C}, S)$. This contradiction proves that radius $\mathscr{X}=\infty$.

Now we can show the essential part of Theorem 3.3(2)(3).

Theorem 4.10. Let $R$ be a local ring. Let $\mathscr{X}$ be a resolving subcategory of $\bmod R$. One has radius $\mathscr{X}=\infty$ if there exists a module $M \in \mathscr{X}$ with $0<\operatorname{Gdim}_{R} M<\infty$ that satisfies either of the following conditions.

(1) $\Omega^{-2} \Omega^{g} M \in \mathscr{X}$, where $g=\operatorname{Gdim}_{R} M$.

(2) $\operatorname{res}\left(\Omega^{n} M\right)$ is a thick subcategory of $\mathscr{G}(R)$ for some $n \geq 0$.

Proof. According to Proposition 4.9, it suffices to show that $\mathscr{X}$ contains a module of projective dimension one.

(1) We consider a construction whose idea essentially comes from the AuslanderBuchweitz approximation theorem [1989, Theorem 1.1]. There are exact sequences $0 \rightarrow \Omega^{g} M \rightarrow R^{\oplus a} \rightarrow \Omega^{g-1} M \rightarrow 0$ and $0 \rightarrow \Omega^{g} M \rightarrow R^{\oplus b} \rightarrow \Omega^{-1} \Omega^{g} M \rightarrow 0$, where the latter is possible as $\Omega^{g} M$ is a totally reflexive module. We make the following pushout diagram.

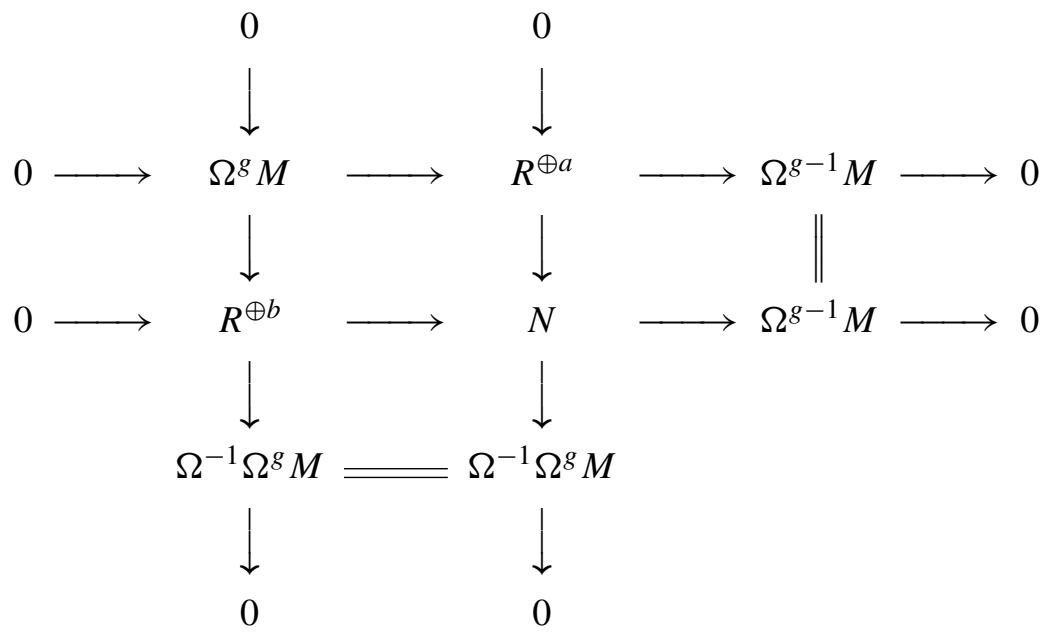

As $\Omega^{-1} \Omega^{g} M$ is totally reflexive, we have $\operatorname{Ext}_{R}^{1}\left(\Omega^{-1} \Omega^{g} M, R\right)=0$. Hence the second column in the above diagram splits, and we get an exact sequence

$$
0 \rightarrow R^{\oplus b} \rightarrow R^{\oplus a} \oplus \Omega^{-1} \Omega^{g} M \rightarrow \Omega^{g-1} M \rightarrow 0 .
$$

There is an exact sequence $0 \rightarrow \Omega^{-1} \Omega^{g} M \rightarrow R^{\oplus c} \rightarrow \Omega^{-2} \Omega^{g} M \rightarrow 0$, and taking the direct sum with $0 \rightarrow R^{\oplus a} \stackrel{=}{\rightarrow} R^{\oplus a} \rightarrow 0 \rightarrow 0$, we have an exact sequence $0 \rightarrow R^{\oplus a} \oplus \Omega^{-1} \Omega^{g} M \rightarrow R^{\oplus(a+c)} \rightarrow \Omega^{-2} \Omega^{g} M \rightarrow 0$. Thus the following pushout 
diagram is obtained.

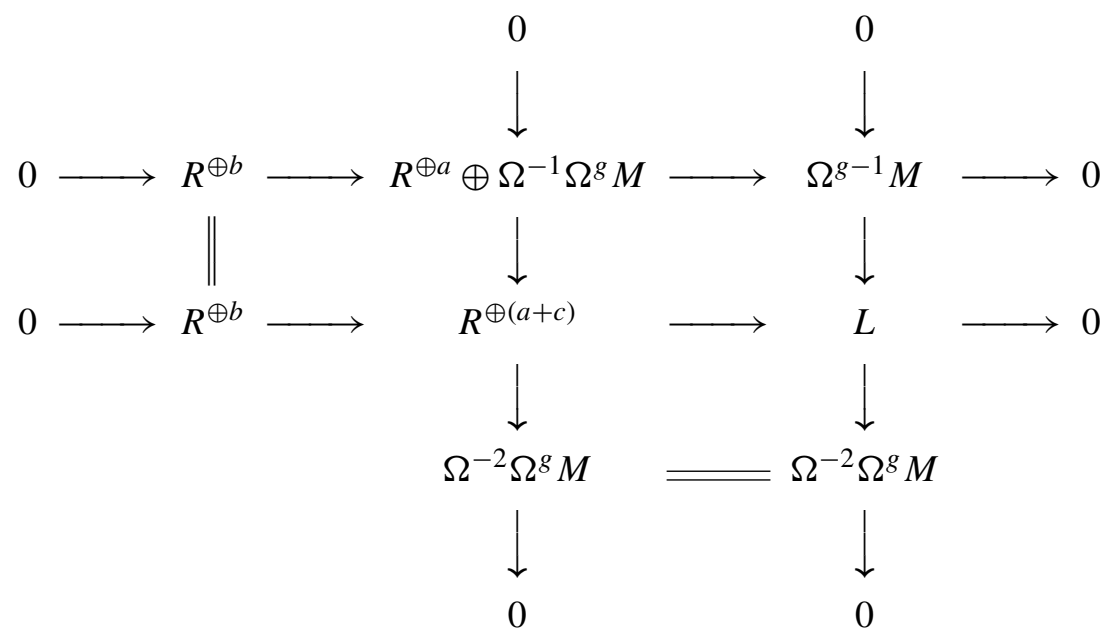

As $\Omega^{g-1} M$ and $\Omega^{-2} \Omega^{g} M$ are in $\mathscr{X}$, the module $L$ is also in $\mathscr{X}$. The second row shows that $L$ has projective dimension at most 1 . Since $\Omega^{-2} \Omega^{g} M$ is totally reflexive but $\Omega^{g-1} M$ is not, it follows from the second column that $L$ is nonfree. Therefore the projective dimension of $L$ is equal to 1 .

(2) Since by assumption res $\Omega^{n} M$ is a subcategory of $\mathscr{G}(R)$, the module $\Omega^{n} M$ is totally reflexive. Hence $n \geq g:=\operatorname{Gdim}_{R} M$.

We claim that res $\Omega^{n} M=$ res $\Omega^{g} M$. In fact, since $n-g \geq 0$ and $\Omega^{n} M=$ $\Omega^{n-g}\left(\Omega^{g} M\right)$, we observe that res $\Omega^{n} M$ is contained in res $\Omega^{g} M$. There is an exact sequence

$$
0 \rightarrow \Omega^{n} M \rightarrow F_{n-1} \rightarrow F_{n-2} \rightarrow \cdots \rightarrow F_{g+1} \rightarrow F_{g} \rightarrow \Omega^{g} M \rightarrow 0
$$

of totally reflexive $R$-modules with each $F_{i}$ being free. Since res $\Omega^{n} M$ is assumed to be thick in $\mathscr{G}(R)$, decomposing the above exact sequence into short exact sequences of totally reflexive modules, we see that $\Omega^{g} M$ belongs to res $\Omega^{n} M$. Therefore res $\Omega^{g} M$ is contained in res $\Omega^{n} M$. Thus the claim follows.

Set $\mathscr{X}=$ res $\Omega^{n} M=$ res $\Omega^{g} M$. Our assumption implies that $\mathscr{X}$ is closed under cosyzygies, whence $\Omega^{-2} \Omega^{g} M \in \mathscr{X}$. By (1), we conclude that $\mathscr{X}$ contains a module of projective dimension 1 .

Now, using Remark 4.1 and Theorem 4.10, we deduce Theorem 3.3(2)(3).

Proof of Theorem 3.3(4). We use the notion of a module of reducible complexity, which has been introduced by Bergh [2007]. Let us recall the definition.

Definition 4.11. The subcategory $\mathscr{C}_{R}^{r}$ of $\bmod R$ is defined inductively as follows.

(1) Every module of finite projective dimension belongs to $\mathscr{C}_{R}^{r}$. 
(2) A module $M$ with $0<\mathrm{cx}_{R} M<\infty$ belongs to $\mathscr{C}_{R}^{r}$ if there exists a homogeneous element $\eta \in \operatorname{Ext}_{R}^{*}(M, M)$ with $|\eta|>0$ which is represented by a short exact sequence $0 \rightarrow M \rightarrow K \rightarrow \Omega^{|\eta|-1} M \rightarrow 0$ with $K \in \mathscr{C}_{R}^{r}$, cx $K<\mathrm{cx} M$ and depth $K=\operatorname{depth} M$.

An $R$-module is said to have reducible complexity if it is in $\mathscr{C}_{R}^{r}$.

The result below is shown in [Bergh 2007, Proposition 2.2(i)], which is implicitly stated in [Avramov et al. 1997].

Proposition 4.12. Let $R$ be a local ring. Every $R$-module of finite CI-dimension has reducible complexity.

In a resolving subcategory, for any fixed integer $n \geq 0$, existence of modules of CI-dimension $n$ is equivalent to existence of modules of projective dimension $n$.

Lemma 4.13. Let $R$ be a local ring. Let $\mathscr{L}$ be a resolving subcategory of $\bmod R$. Suppose that there is a module $M \in \mathscr{X}$ such that $\operatorname{Cldim}_{R} M<\infty$. Then $\mathscr{X}$ contains a module $N$ with $\mathrm{pd}_{R} N=\mathrm{Cldim}_{R} M$.

Proof. Since $M$ has finite CI-dimension, it has finite complexity. It follows from Proposition 4.12 that $M$ has reducible complexity. If cx $M=0$, then $\mathrm{pd} M<\infty$, and we can take $N:=M$. Hence we may assume $\mathrm{cx} M>0$. There exists an exact sequence $0 \rightarrow M \rightarrow K \rightarrow \Omega_{R}^{|\eta|-1} M \rightarrow 0$ with cx $K<\mathrm{cx} M$ and depth $K=\operatorname{depth} M$, where $\eta$ is a homogeneous element of $\operatorname{Ext}_{R}^{*}(M, M)$. We have $K \in \mathscr{L}$ and

Cldim $K=$ depth $R-\operatorname{depth} K=\operatorname{depth} R-\operatorname{depth} M=\operatorname{Cldim} M$.

Replacing $M$ with $K$ and iterating this procedure, we can eventually arrive at a module $N \in \mathscr{X}$ with $C \operatorname{ldim} N=\operatorname{Cldim} M$ and $\operatorname{cx} N=0$. The module $N$ has finite projective dimension, and we have $\operatorname{pd} N=\operatorname{Cldim} N=\operatorname{Cldim} M$.

Lemma 4.13 and Proposition 4.9 immediately yield the following theorem. This is not only the essential part of Theorem 3.3(4) but also a generalization of Proposition 4.9.

Theorem 4.14. Let $R$ be a local ring. Let $\mathscr{X}$ be a resolving subcategory of $\bmod R$. Suppose that there exists a module $M \in \mathscr{X}$ with $0<\operatorname{Cldim}_{R} M<\infty$. Then the radius of $\mathscr{X}$ is infinite.

Theorem 3.3(4) now follows from Theorem 4.14 and Remark 4.1.

Another proof of Theorem 4.14. In the next theorem, we study the thickness of resolving subcategories of modules of CI-dimension at most zero. This will give another proof of Theorem 4.14.

Theorem 4.15. Let $R$ be a local ring. 
(1) Let $M$ be an R-module of CI-dimension at most zero. Then $\Omega^{-1} M$ belongs to res $M$.

(2) Let $\mathscr{L}$ be a resolving subcategory of $\bmod R$. Suppose that every module in $\mathscr{X}$ has CI-dimension at most zero. Then $\mathscr{X}$ is a thick subcategory of $\mathscr{G}(R)$.

Proof. (1) Proposition 4.12 implies that $M$ has reducible complexity. Let $K_{0}=M$ and let $K_{i+1}$ be a reduction in complexity of $K_{i}$ for each $i \geq 0$. Then we have a short exact sequence $0 \rightarrow K_{i} \stackrel{f_{i}}{\rightarrow} K_{i+1} \rightarrow \Omega^{t_{i}-1} K_{i} \rightarrow 0$ with $t_{i}>0$ (see also [Avramov et al. 1997, Proposition 7.2]), and eventually we must have $\mathrm{cx}_{R} K_{e}=0$ for some $e \geq 0$. Then $K_{e}$ has finite projective dimension. As $\operatorname{Cldim}_{R} M=0$, we have depth $K_{e}=$ depth $M=$ depth $R$. Therefore $K_{e}$ is a free module. Note that the above exact sequence also shows that

$$
K_{i} \in \operatorname{res} M \text { for all } i \geq 0 .
$$

If $e=0$, then $M$ is free and we have $\Omega^{-1} M=0 \in \operatorname{res} M$. So we may assume $e \geq 1$.

We claim that for each $0 \leq i \leq e-1$ the cokernel $C_{i}$ of the composite map $f_{i} \cdots f_{1} f_{0}: M \rightarrow K_{i+1}$ belongs to res $M$. Let us show this claim by induction on $i$. When $i=0$, we have $C_{i}=\Omega^{t_{0}-1} M \in$ res $M$. Let $i \geq 1$. We have the following commutative diagram with exact rows and columns.

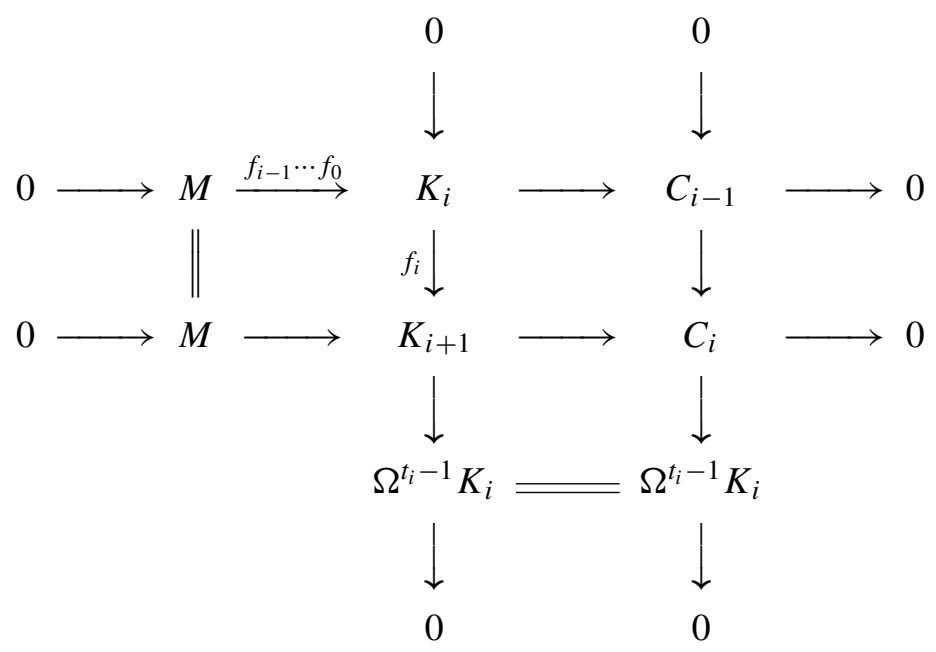

The induction hypothesis implies that $C_{i-1}$ belongs to res $M$. Since $\Omega^{t_{i}-1} K_{i}$ is in res $M$ by (4.15.1), the right column shows that $C_{i}$ is also in res $M$. Thus the claim follows.

Now we have a short exact sequence $0 \rightarrow M \stackrel{f_{e-1} \cdots f_{1} f_{0}}{\longrightarrow} K_{e} \rightarrow C_{e-1} \rightarrow 0$, where $K_{e}$ is free and $C_{e-1}$ is in res $M$ by the claim. Since $M$ is totally reflexive and $\mathscr{G}(R)$ is a resolving subcategory of $\bmod R$, all the modules in res $M$ are totally reflexive. Hence all modules appearing in the above exact sequence belong to $\mathscr{G}(R)$. It is 
easy to verify that there exists an isomorphism $C_{e-1} \cong \Omega^{-1} M \oplus F$ with $F$ being free. Consequently, $\Omega^{-1} M$ belongs to res $M$.

(2) By assumption, $\mathscr{X}$ is a subcategory of $\mathscr{G}(R)$. Thanks to Proposition 1.7 , it is enough to show that $\mathscr{X}$ is closed under cosyzygies. Let $M$ be an $R$-module in $\mathscr{X}$. Then $M$ is of CI-dimension at most zero, and $\Omega^{-1} M$ belongs to res $M$ by (1). Since $\mathscr{X}$ is resolving and contains $M$, it also contains res $M$. Thus $\Omega^{-1} M$ is in $\mathscr{X}$.

Theorem 4.15(2) immediately implies:

Corollary 4.16. Let $R$ be a local complete intersection. The following two are the same.

- A resolving subcategory of $\bmod R$ contained in $\mathrm{CM}(R)$.

- A thick subcategory of $\mathrm{CM}(R)$ containing $R$.

Now let us give another proof of Theorem 4.14. Let $R$ be a local ring, and let $M \in \mathscr{X}$ be an $R$-module with $0<\operatorname{Cldim}_{R} M<\infty$. Then we have $c:=\operatorname{Cldim}_{R} M=$ $\operatorname{Gdim}_{R} M$, and $\Omega^{c} M$ has CI-dimension zero. In particular, $\Omega^{c} M$ is totally reflexive, and hence so is $\Omega^{-1} \Omega^{c} M$. We have

$$
0=\operatorname{Cldim}_{R}\left(\Omega^{c} M\right)=\sup \left\{\operatorname{Cldim}_{R}\left(\Omega^{-1} \Omega^{c} M\right)-1,0\right\},
$$

which especially says that $\Omega^{-1} \Omega^{c} M$ has finite CI-dimension. Therefore

$$
\operatorname{Cldim}_{R}\left(\Omega^{-1} \Omega^{c} M\right)=\operatorname{Gdim}_{R}\left(\Omega^{-1} \Omega^{c} M\right)=0,
$$

and Theorem 4.15(1) yields $\Omega^{-2} \Omega^{c} M \in \operatorname{res}\left(\Omega^{c} M\right) \subseteq \mathscr{X}$. Now Theorem 4.10(1) implies that the radius of $\mathscr{X}$ is infinite.

\section{Proof of Theorem II}

In this section we prove the main Theorem II from the introduction. In fact, we can prove significantly more general statements (Theorems 5.7 and 5.11). In order to state and prove such results we need to first introduce a couple of definitions related to the concept of radius. In this section, an $n$-th syzygy $\Omega^{n} M$ of an $R$-module $M$ means the image of the $n$-th differential map in a projective resolution of $M$ in $\bmod R$. (So it is not unique up to isomorphism but unique up to projective summands.)

Definition 5.1. Let $\mathscr{X}, \mathscr{Y}$ be subcategories of $\bmod R$. We put $|\mathscr{X}|=\operatorname{add} \mathscr{X}$, and set $\mathscr{X} * \mathscr{Y}=|| \mathscr{X}|\circ| \mathscr{Y}||$. (The notation "॰” was introduced in Definition 2.1.) For an integer $r>0$, set

$$
|\mathscr{X}|_{r}= \begin{cases}|\mathscr{X}| & \text { if } r=1, \\ |\mathscr{X}|_{r-1} * \mathscr{X} & \text { if } r \geq 2 .\end{cases}
$$


Let $\mathscr{X}, \mathscr{Y}, \mathscr{L}$ be subcategories of $\bmod R$. We observe that an object $M \in \bmod R$ is in $\mathscr{X} * \mathscr{Y}$ if and only if there is an exact sequence $0 \rightarrow X \rightarrow E \rightarrow Y \rightarrow 0$ with $X \in|\mathscr{X}|$ and $Y \in|\mathscr{Y}|$ such that $M$ is a direct summand of $E$. Also, one has $(\mathscr{X} * \mathscr{Y}) * \mathscr{L}=\mathscr{X} *(\mathscr{Y} * \mathscr{L})$ and $|\mathscr{X}|_{a} *|\mathscr{X}|_{b}=|\mathscr{X}|_{a+b}$ for all $a, b>0$.

Definition 5.2. For a subcategory $\mathscr{X}$ of $\bmod R$ we define size $\mathscr{X}$ (respectively, rank $\mathscr{C}$ ) to be the infimum of integers $n \geq 0$ such that $\mathscr{X} \subseteq|G|_{n+1}$ (respectively, $\mathscr{X}=|G|_{n+1}$ ) for some $G \in \bmod R$.

It always holds that size $\mathscr{X} \leq$ rank $\mathscr{X}$. Since $|\mathscr{X}|_{n} \subseteq[\mathscr{X}]_{n}$ for all $n>0$, one has $\operatorname{dim} \mathscr{X} \geq$ radius $\mathscr{X} \leq$ size $\mathscr{X}$. If $\mathscr{X}$ is resolving, then $\operatorname{dim} \mathscr{X} \leq$ rank $\mathscr{X}$.

For an $R$-module $M$, we denote by $M^{\oplus}$ an object in $\operatorname{add}_{R} M$.

Proposition 5.3. Let $I$ be an ideal of $R$ and let $M$ be an $R / I$-module.

(1) There is an exact sequence $0 \rightarrow I^{\oplus} \rightarrow \Omega_{R} M \rightarrow \Omega_{R / I} M \rightarrow 0$.

(2) One has $\Omega_{R}^{n} M \in\left|\Omega_{R / I}^{n} M \oplus\left(\bigoplus_{i=0}^{n-1} \Omega_{R}^{i} I\right)\right|_{n+1}$ for all $n \geq 0$.

Proof. (1) Take a surjection from a free $R$-module $F$ to $M$. Then this factors through a surjection $F / I F \rightarrow M$. The assertion follows from this.

(2) We induce on $n$. Let $n>0$. The induction hypothesis shows

$$
\Omega_{R}^{n-1} \Omega_{R / I} M \in\left|\Omega_{R / I}^{n} M \oplus\left(\bigoplus_{i=0}^{n-2} \Omega_{R}^{i} I\right)\right|_{n} .
$$

By (1) we have an exact sequence $0 \rightarrow \Omega_{R}^{n-1} I^{\oplus} \rightarrow \Omega_{R}^{n} M \rightarrow \Omega_{R}^{n-1} \Omega_{R / I} M \rightarrow 0$. Now the assertion follows.

For $n \geq 0$ we denote by $\Omega_{R}^{n}(\bmod R)$ the subcategory of $\bmod R$ consisting of $n$-th syzygies of $R$-modules. For an ideal $I$ of $R$, let $\Omega_{R}^{n}(\bmod R / I)$ be the subcategory of $\bmod R$ consisting of $n$-th syzygies of $R$-modules annihilated by $I$.

Corollary 5.4. Let $d=\operatorname{dim} R<\infty$. Suppose that $R / \mathfrak{p}$ is regular for all $\mathfrak{p} \in \operatorname{Min} R$. Then size $\Omega^{d}(\bmod R)<\infty$.

Proof. There is a filtration $R=I_{0} \supsetneq I_{1} \supsetneq \cdots \supsetneq I_{n}=0$ of ideals of $R$ such that for each $i$ one has $I_{i} / I_{i+1} \cong R / \mathfrak{p}_{i}$ with $\mathfrak{p}_{i} \in \operatorname{Spec} R$. Choose a minimal prime $\mathfrak{q}_{i}$ contained in $\mathfrak{p}_{i}$. Let $M$ be an $R$-module. Setting $M_{i}=I_{i} M / I_{i+1} M$, we have an exact sequence $0 \rightarrow \Omega_{R}^{d}\left(I_{i+1} M\right) \rightarrow \Omega_{R}^{d}\left(I_{i} M\right) \rightarrow \Omega_{R}^{d} M_{i} \rightarrow 0$. Note that each $d$-th syzygy $R / \mathfrak{q}_{i}$-module is free. Hence $\Omega_{R}^{d} M_{i} \in\left|R / \mathfrak{q}_{i} \oplus L_{i}\right|_{d+1}$ by Proposition 5.3(2), where $L_{i}:=\bigoplus_{j=0}^{d-1} \Omega_{R}^{j} \mathfrak{q}_{i}$. Thus $\Omega_{R}^{d} M \in\left|\bigoplus_{i=1}^{n}\left(R / \mathfrak{q}_{i} \oplus L_{i}\right)\right|_{n(d+1)}$, which implies size $\Omega^{d}(\bmod R)<n(d+1)<\infty$.

Corollary 5.5. Let $I$ be an ideal of $R$ and $n \geq 0$ an integer. Then size $\Omega_{R}^{n}(\bmod R / I)<(n+1)\left(\operatorname{size} \Omega_{R / I}^{n}(\bmod R / I)+1\right)$. 
In particular, if size $\Omega_{R / I}^{n}(\bmod R / I)$ is finite, then so is size $\Omega_{R}^{n}(\bmod R / I)$.

Proof. This is a consequence of Proposition 5.3(2).

Lemma 5.6. Let $M$ be an $R$-module. Let $x \in R$ be $R$-regular. Then

$$
\Omega_{R / x R}^{n}\left(\Omega_{R} M / x \Omega_{R} M\right) \cong \Omega_{R}^{n+1} M / x \Omega_{R}^{n+1} M
$$

for any $n \geq 0$.

Proof. We use induction on $n$. Let $n>0$. We have

$$
\Omega_{R / x R}^{n-1}(\Omega M / x \Omega M) \cong \Omega^{n} M / x \Omega^{n} M
$$

by the induction hypothesis, and hence

$$
\Omega_{R / x R}^{n}(\Omega M / x \Omega M) \cong \Omega_{R / x R}\left(\Omega^{n} M / x \Omega^{n} M\right) .
$$

Note that $x$ is $\Omega_{R}^{n} M$-regular. There is an exact sequence

$$
0 \rightarrow \Omega^{n+1} M \rightarrow P \rightarrow \Omega^{n} M \rightarrow 0
$$

of $R$-modules with $P$ projective, which gives an exact sequence

$$
0 \rightarrow \Omega^{n+1} M / x \Omega^{n+1} M \rightarrow P / x P \rightarrow \Omega^{n} M / x \Omega^{n} M \rightarrow 0 .
$$

Hence $\Omega^{n+1} M / x \Omega^{n+1} M \cong \Omega_{R / x R}\left(\Omega^{n} M / x \Omega^{n} M\right)$.

Theorem 5.7. Let $(R, \mathfrak{m})$ be a d-dimensional complete local ring with perfect coefficient field. Then one has size $\Omega^{d}(\bmod R)<\infty$. Hence radius $\Omega^{d}(\bmod R)<\infty$.

Proof. We use induction on $d$. When $d=0$, we have $\bmod R=|k|_{\ell \ell(R)}$, hence size $(\bmod R)<\ell \ell(R)<\infty$. Let $d \geq 1$. Take a filtration $R=I_{0} \supsetneq \cdots \supsetneq I_{n}=0$ of ideals such that for each $i$ one has $I_{i} / I_{i+1} \cong R / \mathfrak{p}_{i}$ with $\mathfrak{p}_{i} \in \operatorname{Spec} R$. Suppose that size $\Omega_{R / \mathfrak{p}_{i}}^{d_{i}}\left(\bmod R / \mathfrak{p}_{i}\right)<\infty$ for all $i$, where $d_{i}=\operatorname{dim} R / \mathfrak{p}_{i}$. Then we have

$$
\operatorname{size} \Omega_{R / \mathfrak{p}_{i}}^{d}\left(\bmod R / \mathfrak{p}_{i}\right)<\infty,
$$

since $\Omega_{R / \mathfrak{p}_{i}}^{d}\left(\bmod R / \mathfrak{p}_{i}\right)$ is contained in $\Omega_{R / \mathfrak{p}_{i}}^{d_{i}}\left(\bmod R / \mathfrak{p}_{i}\right)$. Corollary 5.5 implies size $\Omega_{R}^{d}\left(\bmod R / \mathfrak{p}_{i}\right)<\infty$. For each $R$-module $M$ there is an exact sequence $0 \rightarrow I_{i+1} M \rightarrow I_{i} M \rightarrow I_{i} M / I_{i+1} M \rightarrow 0$, which gives an exact sequence

$$
0 \rightarrow \Omega_{R}^{d}\left(I_{i+1} M\right) \rightarrow \Omega_{R}^{d}\left(I_{i} M\right) \rightarrow \Omega_{R}^{d}\left(I_{i} M / I_{i+1} M\right) \rightarrow 0 .
$$

As $\Omega_{R}^{d}\left(I_{i} M / I_{i+1} M\right)$ is in $\Omega_{R}^{d}\left(\bmod R / \mathfrak{p}_{i}\right)$, we have size $\Omega_{R}^{d}(\bmod R)<\infty$. Thus, we may assume $R$ is a domain.

If $R$ is regular, then $\Omega^{d}(\bmod R)=|R|_{1}$ and size $\Omega^{d}(\bmod R)=0<\infty$, so we may assume that $R$ is singular. By [Wang 1994, 5.15] there is an ideal $J \subseteq \mathfrak{m}$ with 
Sing $R=\mathrm{V}(J)$ and $J \operatorname{Ext}_{R}^{d+1}(\bmod R, \bmod R)=0$. Since $R$ is a domain, we find an element $0 \neq x \in J$. The induction hypothesis guarantees

$$
\Omega_{R / x R}^{d-1}(\bmod R / x R) \subseteq|G|_{n}^{R / x R}
$$

for some $R / x R$-module $G$ and an integer $n>0$. Let $M$ be an $R$-module and put $N=\Omega_{R}^{d} M$. Note that $x$ is $N$-regular as $d>0$. Hence $N$ is isomorphic to a direct summand of $\Omega_{R}(N / x N)$ (see [Takahashi 2010, Lemma 2.1]). In view of Lemma 5.6, we have

$$
N / x N \cong \Omega_{R / x R}^{d-1}\left(\Omega_{R} M / x \Omega_{R} M\right) \in \Omega_{R / x R}^{d-1}(\bmod R / x R) \subseteq|G|_{n}^{R / x R} .
$$

Hence $N / x N$ is in $|G|_{n}^{R}$, which implies $\Omega_{R}(N / x N) \in\left|\Omega_{R} G\right|_{n}^{R}$. Therefore $N$ belongs to $\left|\Omega_{R} G\right|_{n}^{R}$, and we obtain $\Omega^{d}(\bmod R) \subseteq\left|\Omega_{R} G\right|_{n}^{R}$. It now follows that size $\Omega^{d}(\bmod R)<\infty$.

Lemma 5.8. Let

$$
0 \rightarrow M \rightarrow C^{0} \rightarrow C^{1} \rightarrow \cdots \rightarrow C^{n-1} \rightarrow N \rightarrow 0
$$

be an exact sequence in $\bmod R$ with $n \geq 0$. Then $M$ is in $\left|\Omega^{n} N \oplus\left(\bigoplus_{i=0}^{n-1} \Omega^{i} C^{i}\right)\right|_{n+1}$. Proof. We induce on $n$. The case $n=0$ is trivial, so let $n \geq 1$. There are two exact sequences $0 \rightarrow M \rightarrow C^{0} \rightarrow L \rightarrow 0$ and $0 \rightarrow L \rightarrow C^{1} \rightarrow \cdots \rightarrow C^{n-1} \rightarrow N \rightarrow 0$. The induction hypothesis shows $L \in\left|\Omega^{n-1} N \oplus\left(\bigoplus_{i=0}^{n-2} \Omega^{i} C^{i+1}\right)\right|_{n}$. A pullback diagram makes an exact sequence $0 \rightarrow \Omega L \rightarrow M \oplus R^{\oplus} \rightarrow C^{0} \rightarrow 0$. Since $\Omega L$ belongs to $\left|\Omega^{n} N \oplus\left(\bigoplus_{i=0}^{n-2} \Omega^{i+1} C^{i+1}\right)\right|_{n}$, we see that $M$ is in $\left|\Omega^{n} N \oplus\left(\bigoplus_{i=0}^{n-1} \Omega^{i} C^{i}\right)\right|_{n+1}$.

Corollary 5.9. Let $R$ be a Cohen-Macaulay complete local ring with perfect coefficient field. Then size $\mathrm{CM}(R)<\infty$.

Proof. As $R$ is complete, it admits a canonical module $\omega$. Theorem 5.7 implies size $\Omega^{d}(\bmod R)=: n<\infty$, so we have $\Omega^{d}(\bmod R) \subseteq|G|_{n+1}$ for some $R$-module $G$. Let $M$ be a Cohen-Macaulay $R$-module. Then there exists an exact sequence $0 \rightarrow M \rightarrow \omega^{\oplus s_{0}} \rightarrow \cdots \rightarrow \omega^{\oplus s_{d-1}} \rightarrow N \rightarrow 0$. It follows from Lemma 5.8 that $M$ is in $\left|\Omega^{d} N \oplus W\right|_{d+1}$, where $W:=\bigoplus_{i=0}^{d-1} \Omega^{i} \omega$. Since $\Omega^{d} N \in|G|_{n+1}$, we have $M \in|G \oplus W|_{(n+1)(d+1)}$. Thus size $\operatorname{CM}(R)<(n+1)(d+1)<\infty$.

Proposition 5.10. Let $R$ be a Cohen-Macaulay local ring with a canonical module $\omega$.

(1) $\operatorname{rank} \mathrm{CM}(R)<(\operatorname{dim} R+1)(\operatorname{size} \mathrm{CM}(R)+1)$.

(2) $\operatorname{dim} \mathrm{CM}(R)<(\operatorname{dim} R+1)($ radius $\mathrm{CM}(R)+1)$.

In particular, one has

rank $\mathrm{CM}(R)<\infty \Leftrightarrow \operatorname{size} \mathrm{CM}(R)<\infty \Rightarrow \operatorname{dim} \mathrm{CM}(R)<\infty \Leftrightarrow$ radius $\mathrm{CM}(R)<\infty$. 
Proof. (1) Let $n=$ size $\mathrm{CM}(R)$. We find an $R$-module $G$ with $\mathrm{CM}(R) \subseteq|G|_{n+1}$. Let $d=\operatorname{dim} R$ and $M \in \mathrm{CM}(R)$. Similarly to the proof of Corollary 5.9, there exists $N \in \mathrm{CM}(R)$ such that $M$ is in $\left|\Omega^{d} N \oplus W\right|_{d+1}$, where $W:=\bigoplus_{i=0}^{d-1} \Omega^{i} \omega \in \mathrm{CM}(R)$. Note that $\Omega^{d} N \in\left|\Omega^{d} G\right|_{n+1}$. Thus we obtain

$$
\mathrm{CM}(R)=\left|\Omega^{d} G \oplus W\right|_{(n+1)(d+1)},
$$

and $\operatorname{rank} \mathrm{CM}(R)<(n+1)(d+1)$.

(2) In the proof of (1), replace "size", "rank" and "I |" with "radius", "dim" and "[ ]", respectively.

Theorem 5.11. Let $R$ be a Cohen-Macaulay local ring admitting perfect coefficient field. Assume that either of the following holds.

(1) $R$ is complete.

(2) $R$ is excellent with an isolated singularity.

Then one has rank $\mathrm{CM}(R)<\infty$.

Proof. (1) This assertion follows from Corollary 5.9 and Proposition 5.10(1).

(2) It follows by (1) that $\mathrm{CM}(\widehat{R})$ has finite rank, where $\widehat{R}$ denotes the completion of $R$. One can prove that $\mathrm{CM}(R)$ also has finite rank, making an argument similar to [Dao and Takahashi 2012, Remark 6.5]:

Putting $n=\operatorname{rank} \operatorname{CM}(\widehat{R})$, we have $\operatorname{CM}(\widehat{R})=|C|_{n+1}$ for some $C \in \mathrm{CM}(\widehat{R})$. Since $R$ is Cohen-Macaulay, excellent and with an isolated singularity, we can apply [Takahashi 2010, Corollary 3.6] to see that $C$ is isomorphic to a direct summand of $\widehat{G}$ for some $G \in \operatorname{CM}(R)$. Hence the equality $\operatorname{CM}(\widehat{R})=|\widehat{G}|_{n+1}$ holds.

Claim. Let $m>0$. For any $N \in|\widehat{G}|_{m}$ there exists $M \in|G|_{m}$ such that $N$ is isomorphic to a direct summand of $\widehat{M}$.

To show this claim, we use induction on $m$. As $R$ is an isolated singularity, for all $X, Y \in \mathrm{CM}(R)$ the $R$-module $\operatorname{Ext}_{R}^{1}(X, Y)$ has finite length. Hence there are isomorphisms $\operatorname{Ext}_{R}^{1}(X, Y) \cong \operatorname{Ext}_{R}^{1}(X, Y) \cong \operatorname{Ext}_{\widehat{R}}^{1}(\widehat{X}, \widehat{Y})$, which imply that every short exact sequence $0 \rightarrow \widehat{Y} \rightarrow E \rightarrow \widehat{X} \rightarrow 0$ of $\widehat{R}$-modules is isomorphic to the completion of some short exact sequence $0 \rightarrow Y \rightarrow E^{\prime} \rightarrow X \rightarrow 0$ of $R$-modules. The claim follows from this. Using this claim and [Aihara and Takahashi 2011, Lemma 5.7], we observe that $\mathrm{CM}(R)=|G|_{n+1}$ holds. Therefore rank $\mathrm{CM}(R) \leq n<\infty$.

\section{Some discussions and open questions}

In this section we relate our results to the uniform Auslander condition and discuss some open questions. For a local ring $R$, Jorgensen and Şega [2004] introduced the uniform Auslander condition: 
(UAC): There exists an integer $n$ such that for all $R$-modules $M, N$ with $\operatorname{Ext}_{R}^{i}(M, N)=0$ for all $i \gg 0$ one has $\operatorname{Ext}_{R}^{i}(M, N)=0$ for all $i \geq n$.

It is known that this condition is satisfied if the local ring $R$ is a complete intersection, a Golod ring, a Gorenstein ring with mult $R=\operatorname{codim} R+2$, or a Gorenstein ring with codim $R \leq 4$. Here mult $R$ denotes the multiplicity of $R$. These are proved in [Jorgensen and Şega 2004, Proposition 1.4], [Avramov and Buchweitz 2000, Theorem 4.7], [Huneke and Jorgensen 2003, Theorem 3.5] and [Şega 2003, Theorem 3.4], respectively. More information can be found in [Christensen and Holm 2010, Appendix A]. On the other hand, there exists an example of a Gorenstein local ring which does not satisfy (UAC); see [Jorgensen and Şega 2004, Theorem in §0].

The result below says that over a Gorenstein local ring the condition (UAC) is closely related to the thickness of resolving subcategories of Cohen-Macaulay modules.

Proposition 6.1. Let $R$ be a Gorenstein local ring. Assume every resolving subcategory of $\bmod R$ contained in $\mathrm{CM}(R)$ is a thick subcategory of $\mathrm{CM}(R)$. Then $R$ satisfies (UAC).

Proof. Let $t \geq 0$ be an integer, and let $M, N$ be $R$-modules with $\operatorname{Ext}_{R}^{i}(M, N)=0$ for all $i>t$. We define a subcategory $\mathscr{L}$ of $\bmod R$ to consist of all Cohen-Macaulay $R$-modules $X$ satisfying $\operatorname{Ext}_{R}^{i}(X, N)=0$ for all $i>t$. Then $\mathscr{L}$ is a resolving subcategory of $\bmod R$ contained in $\mathrm{CM}(R)$. By assumption, $\mathscr{X}$ is a thick subcategory of $\operatorname{CM}(R)$. Set $d=\operatorname{dim} R$. Since $\Omega^{d} M$ is in $\mathscr{L}$, so is $\Omega^{-t} \Omega^{d} M$. We have

$$
\begin{aligned}
\operatorname{Ext}_{R}^{i}(M, N) & \cong \operatorname{Ext}_{R}^{i-d}\left(\Omega^{d} M, N\right) \cong \operatorname{Ext}_{R}^{i-d}\left(\Omega^{t}\left(\Omega^{-t} \Omega^{d} M\right), N\right) \\
& \cong \operatorname{Ext}_{R}^{i-d+t}\left(\Omega^{-t} \Omega^{d} M, N\right)=0
\end{aligned}
$$

for all integers $i>d$.

There is also a connection between thickness of resolving subcategories of totally reflexive modules and closure under $R$-duals. Here we say that a subcategory $\mathscr{X}$ of mod $R$ is closed under $R$-duals if for each module $M$ in $\mathscr{X}$ its $R$-dual $M^{*}$ is also in $\mathscr{X}$.

Proposition 6.2. (1) Let $R$ be local. Let $\mathscr{Q}$ be a resolving subcategory of $\bmod R$ contained in $\mathscr{G}(R)$. If $\mathscr{X}$ is closed under $R$-duals, then $\mathscr{X}$ is a thick subcategory of $\mathscr{G}(R)$.

(2) Let $R$ be a local hypersurface. Then every resolving subcategory of $\bmod R$ contained in $\mathrm{CM}(R)$ is closed under $R$-duals.

Proof. (1) According to Proposition 1.7, we have only to show that $\mathscr{X}$ is closed under cosyzygies. Let $X \in \mathscr{X}$. There is an exact sequence $0 \rightarrow \Omega\left(X^{*}\right) \rightarrow F \rightarrow X^{*} \rightarrow 0$, 
where $F$ is free. Dualizing this by $R$, we get an exact sequence

$$
0 \rightarrow X \rightarrow F^{*} \rightarrow\left(\Omega\left(X^{*}\right)\right)^{*} \rightarrow 0 .
$$

Note that $\left(\Omega\left(X^{*}\right)\right)^{*}$ is totally reflexive. We easily see that $\left(\Omega\left(X^{*}\right)\right)^{*}$ is isomorphic to $\Omega^{-1} X$ up to free summand. As $\mathscr{X}$ is a resolving subcategory closed under $R$-duals, $\left(\Omega\left(X^{*}\right)\right)^{*}$ belongs to $\mathscr{X}$, and so does $\Omega^{-1} X$.

(2) It follows from [Takahashi 2010, main theorem] that every resolving subcategory of mod $R$ contained in $\mathrm{CM}(R)$ can be described as $\mathrm{NF}_{\mathrm{CM}}^{-1}(W)$, where $W$ is a specialization-closed subset of Spec $R$ contained in $\operatorname{Sing} R$. If $M$ is an $R$-module in $\mathrm{NF}_{\mathrm{CM}}^{-1}(W)$, then we have $\mathrm{NF}\left(M^{*}\right)=\mathrm{NF}(M) \subseteq W$, which shows that $\mathrm{NF}_{\mathrm{CM}}^{-1}(W)$ also contains $M^{*}$.

Now we have reached the following question.

Question 6.3. Let $R$ be a Gorenstein local ring. Let us consider the following five conditions.

(1) $R$ is a complete intersection.

(2) Every resolving subcategory of $\bmod R$ contained in $\operatorname{CM}(R)$ is closed under $R$-duals.

(3) Every resolving subcategory of $\bmod R$ contained in $\operatorname{CM}(R)$ is a thick subcategory of $\mathrm{CM}(R)$.

(4) $R$ satisfies (UAC).

(5) Conjecture 3.1 is true for $R$.

We know that the implications $(2) \Rightarrow(3)$ and $(1) \Rightarrow(3) \Rightarrow$ (4) hold by Propositions $6.1,6.2(1)$ and Corollary 4.16. The implication $(1) \Rightarrow(2)$ is also true if $R$ is a hypersurface by Proposition 6.2(2). Very recently, motivated by the first version of the present paper, Stevenson [2013a] proved that the implication $(1) \Rightarrow(2)$ holds in the case where $R$ is a quotient of a regular local ring. Corollary 3.4 says that (3) $\Rightarrow$ (5) holds. How about the other implications among these five conditions?

Remark 6.4. According to a recent preprint by Stevenson [2013b] (see also [Iyengar 2009]), if $R$ is a quotient of a regular local ring by a regular sequence, then one can classify the thick subcategories of $\underline{\mathrm{CM}}(R)$ in terms of "support varieties". Thus, one can also classify the resolving subcategories of $\bmod R$ contained in $\operatorname{CM}(R)$ by using Corollary 4.16 and [Takahashi 2010, Proposition 6.2]. In relation to this, the resolving subcategories over a regular ring can be classified completely. This classification theorem is stated and proved in [Dao and Takahashi 2013]. 


\section{Acknowledgments}

We would like to thank Luchezar Avramov, Jesse Burke, Craig Huneke, Osamu Iyama, Srikanth Iyengar and Greg Stevenson for their valuable comments. This work was done during the visits of Takahashi to University of Kansas in May, July and August, 2011, and July, 2012. He is grateful for their kind hospitality. We also thank the referee for a careful reading and helpful comments.

\section{References}

[Aihara and Takahashi 2011] T. Aihara and R. Takahashi, "Generators and dimensions of derived categories", preprint, 2011. arXiv 1106.0205

[Araya et al. 2012] T. Araya, K.-i. Iima, and R. Takahashi, "On the structure of Cohen-Macaulay modules over hypersurfaces of countable Cohen-Macaulay representation type", J. Algebra 361 (2012), 213-224. MR 2921619 Zbl 1275.13009

[Auslander 1967] M. Auslander, Anneaux de Gorenstein, et torsion en algèbre commutative, Secrétariat mathématique, Paris, 1967. MR 37 \#1435 Zbl 0157.08301

[Auslander and Bridger 1969] M. Auslander and M. Bridger, Stable module theory, Memoirs of the American Mathematical Society 94, Amer. Math. Soc., Providence, R.I., 1969. MR 42 \#4580 Zbl 0204.36402

[Auslander and Buchweitz 1989] M. Auslander and R.-O. Buchweitz, "The homological theory of maximal Cohen-Macaulay approximations", pp. 5-37 Mém. Soc. Math. France (N.S.) 38, 1989. MR 91h:13010 Zbl 0697.13005

[Avramov and Buchweitz 2000] L. L. Avramov and R.-O. Buchweitz, "Support varieties and cohomology over complete intersections", Invent. Math. 142:2 (2000), 285-318. MR 2001j:13017 Zbl 0999.13008

[Avramov et al. 1997] L. L. Avramov, V. N. Gasharov, and I. V. Peeva, "Complete intersection dimension”, Inst. Hautes Études Sci. Publ. Math. 86 (1997), 67-114. MR 99c:13033 Zbl 0918.13008

[Avramov et al. 2010] L. L. Avramov, R.-O. Buchweitz, S. B. Iyengar, and C. Miller, "Homology of perfect complexes", Adv. Math. 223:5 (2010), 1731-1781. MR 2011k:13014 Zbl 1186.13006

[Bergh 2007] P. A. Bergh, "Modules with reducible complexity", J. Algebra 310:1 (2007), 132-147. MR 2008g:13021 Zbl 1117.13016

[Buchweitz 1986] R. O. Buchweitz, "Maximal Cohen-Macaulay modules and Tate-cohomology over Gorenstein rings", preprint, 1986, Available at http://hdl.handle.net/1807/16682.

[Christensen 2000] L. W. Christensen, Gorenstein dimensions, Lecture Notes in Mathematics 1747, Springer, Berlin, 2000. MR 2002e:13032 Zbl 0965.13010

[Christensen and Holm 2010] L. W. Christensen and H. Holm, "Algebras that satisfy Auslander's condition on vanishing of cohomology”, Math. Z. 265:1 (2010), 21-40. MR 2011c:16033 Zbl 1252.16008

[Dao and Takahashi 2012] H. Dao and R. Takahashi, "The dimension of a subcategory of modules", preprint, 2012. arXiv 1203.1955

[Dao and Takahashi 2013] H. Dao and R. Takahashi, "Classification of resolving subcategories and grade consistent functions", 2013. To appear in Int. Math. Res. Not.

[Happel 1988] D. Happel, Triangulated categories in the representation theory of finite-dimensional algebras, London Mathematical Society Lecture Note Series 119, Cambridge University Press, 1988. MR 89e:16035 Zbl 0635.16017 
[Huneke and Jorgensen 2003] C. Huneke and D. A. Jorgensen, "Symmetry in the vanishing of Ext over Gorenstein rings”, Math. Scand. 93:2 (2003), 161-184. MR 2004k:13039 Zbl 1062.13005

[Iyengar 2009] S. B. Iyengar, "Stratifying derived categories associated to finite groups and commutative rings, Kyoto RIMS Workshop on Algebraic Triangulated Categories and Related Topics", Lecture notes, 2009, Available at http://www.math.unl.edu/ siyengar2/Papers/RIMS0709.pdf.

[Jorgensen and Şega 2004] D. A. Jorgensen and L. M. Şega, "Nonvanishing cohomology and classes of Gorenstein rings", Adv. Math. 188:2 (2004), 470-490. MR 2005f:13017 Zbl 1090.13009

[Minamoto 2013] H. Minamoto, "A note on dimension of triangulated categories", Proc. Amer. Math. Soc. 141:12 (2013), 4209-4214. MR 3105864 Zbl 06218150

[Rouquier 2008] R. Rouquier, “Dimensions of triangulated categories”, J. K-Theory 1:2 (2008), 193-256. MR 2009i:18008 Zbl 1165.18008

[Şega 2003] L. M. Şega, "Vanishing of cohomology over Gorenstein rings of small codimension", Proc. Amer. Math. Soc. 131:8 (2003), 2313-2323. MR 2004b:13016 Zbl 1017.13008

[Stevenson 2013a] G. Stevenson, "Duality for bounded derived categories of complete intersections", 2013. To appear in Bull. London Math. Soc.

[Stevenson 2013b] G. Stevenson, "Subcategories of singularity categories via tensor actions", 2013. To appear in Compos. Math.

[Takahashi 2009] R. Takahashi, "Modules in resolving subcategories which are free on the punctured spectrum”, Pacific J. Math. 241:2 (2009), 347-367. MR 2010b:13027 Zbl 1172.13005

[Takahashi 2010] R. Takahashi, "Classifying thick subcategories of the stable category of CohenMacaulay modules”, Adv. Math. 225:4 (2010), 2076-2116. MR 2011h:13014 Zbl 1202.13009

[Takahashi 2013] R. Takahashi, "Classifying resolving subcategories over a Cohen-Macaulay local ring”, Math. Z. 273:1-2 (2013), 569-587. MR 3010176 Zbl 1267.13024

[Wang 1994] H.-J. Wang, "On the Fitting ideals in free resolutions", Michigan Math. J. 41:3 (1994), 587-608. MR 96b:13013 Zbl 0822.13007

[Yoshino 2005] Y. Yoshino, "A functorial approach to modules of G-dimension zero", Illinois J. Math. 49:2 (2005), 345-367. MR 2006e:13014 Zbl 1097.13019

[Yoshiwaki 2011] M. Yoshiwaki, “On self-injective algebras of stable dimension zero", Nagoya Math. J. 203 (2011), 101-108. MR 2012h:16009 Zbl 1227.16016

Communicated by Kei-Ichi Watanabe

Received 2012-07-14 Revised 2013-08-10 Accepted 2013-09-14

hdao@math.ku.edu

Department of Mathematics, University of Kansas, 405 Snow Hall, 1460 Jayhawk Blvd, Lawrence, KS 66045, United States http://www.math.ku.edu/ hdao/

takahashi@math.nagoya-u.ac.jp Department of Mathematics, University of Nebraska, Lincoln, NE 68588-0130, United States

Current address: Graduate School of Mathematics, Nagoya University, Furocho, Chikusaku, Nagoya 464-8602, Japan http://www.math.nagoya-u.ac.jp/ takahashi/ 


\section{Algebra \& Number Theory}

msp.org/ant

\section{EDITORS}

MANAGING EDITOR

Bjorn Poonen

Massachusetts Institute of Technology

Cambridge, USA

\author{
EDITORIAL BOARD CHAIR \\ David Eisenbud \\ University of California \\ Berkeley, USA
}

\section{BOARD OF EDITORS}

Georgia Benkart

Dave Benson

Richard E. Borcherds

John H. Coates

J-L. Colliot-Thélène

Brian D. Conrad

Hélène Esnault

Hubert Flenner

Edward Frenkel

Andrew Granville

Joseph Gubeladze

Roger Heath-Brown

Ehud Hrushovski

Craig Huneke

Mikhail Kapranov

Yujiro Kawamata

János Kollár

Yuri Manin

Barry Mazur

Philippe Michel
University of Wisconsin, Madison, USA

University of Aberdeen, Scotland

University of California, Berkeley, USA

University of Cambridge, UK

CNRS, Université Paris-Sud, France

University of Michigan, USA

Freie Universität Berlin, Germany

Ruhr-Universität, Germany

University of California, Berkeley, USA

Université de Montréal, Canada

San Francisco State University, USA

Oxford University, UK

Hebrew University, Israel

University of Virginia, USA

Yale University, USA

University of Tokyo, Japan

Princeton University, USA

Northwestern University, USA

Harvard University, USA

École Polytechnique Fédérale de Lausanne
Susan Montgomery

Shigefumi Mori

Raman Parimala

Jonathan Pila

Victor Reiner

Karl Rubin

Peter Sarnak

Joseph H. Silverman

Michael Singer

Vasudevan Srinivas

J. Toby Stafford

Bernd Sturmfels

Richard Taylor

Ravi Vakil

Michel van den Bergh

Marie-France Vignéras

Kei-Ichi Watanabe

Efim Zelmanov

Shou-Wu Zhang
University of Southern California, USA

RIMS, Kyoto University, Japan

Emory University, USA

University of Oxford, UK

University of Minnesota, USA

University of California, Irvine, USA

Princeton University, USA

Brown University, USA

North Carolina State University, USA

Tata Inst. of Fund. Research, India

University of Michigan, USA

University of California, Berkeley, USA

Harvard University, USA

Stanford University, USA

Hasselt University, Belgium

Université Paris VII, France

Nihon University, Japan

University of California, San Diego, USA

Princeton University, USA

PRODUCTION

production@msp.org

Silvio Levy, Scientific Editor

See inside back cover or msp.org/ant for submission instructions.

The subscription price for 2014 is US $\$ 225 /$ year for the electronic version, and $\$ 400 /$ year $(+\$ 55$, if shipping outside the US) for print and electronic. Subscriptions, requests for back issues and changes of subscribers address should be sent to MSP.

Algebra \& Number Theory (ISSN 1944-7833 electronic, 1937-0652 printed) at Mathematical Sciences Publishers, 798 Evans Hall \#3840, c/o University of California, Berkeley, CA 94720-3840 is published continuously online. Periodical rate postage paid at Berkeley, CA 94704, and additional mailing offices.

ANT peer review and production are managed by EditFLOW ${ }^{\circledR}$ from Mathematical Sciences Publishers.

\section{PUBLISHED BY}

- mathematical sciences publishers

nonprofit scientific publishing

http://msp.org/

(C) 2014 Mathematical Sciences Publishers 


\section{Algebra \& Number Theory}

\section{Volume $8 \quad$ No. $1 \quad 2014$}

On the Picard number of K3 surfaces over number fields

FRANÇOIS CHARLES

Adèle residue symbol and Tate's central extension for multiloop Lie algebras

OLIVER BRAUNLING

On the number of cubic orders of bounded discriminant having automorphism group $C_{3}$, and related problems

MANJUl Bhargava and ARIEl ShNidman

Polynomial bounds for Arakelov invariants of Belyi curves

ARIYAN JAVANPEYKAR

The radius of a subcategory of modules

HAILONG DAO and RYO TAKAHASHI

A generalized Bogomolov-Gieseker inequality for the three-dimensional projective space

EMANUELE MACRÌ

$(\varphi, \Gamma)$-modules over noncommutative overconvergent and Robba rings

GERGELY ZÁBRÁDI

The Tannakian formalism and the Langlands conjectures

DAVID KAZHDAN, MICHAEL LARSEN and YAKOV VARSHAVSKY 